\title{
Cellular metabolic basis of altered immunity in the lungs of patients with COVID-19
}

\author{
Shuangyan $\mathrm{Li}^{1} \cdot$ Fuxiaonan $\mathrm{Zhao}^{1} \cdot$ Jing $\mathrm{Ye}^{1} \cdot \mathrm{Kuan}^{\mathrm{Li}}{ }^{1,2} \cdot \mathrm{Qi} \mathrm{Wang}^{1,2} \cdot \mathrm{Zhongchao} \mathrm{Du}^{1,2} \cdot \mathrm{Qing} \mathrm{Yue}^{1} \cdot$ Sisi Wang ${ }^{1}$. \\ Qi Wu ${ }^{1,2} \cdot$ Huaiyong Chen ${ }^{1,2,3,4}$ (i)
}

Received: 24 September 2021 / Accepted: 27 December 2021 / Published online: 13 January 2022

(c) The Author(s), under exclusive licence to Springer-Verlag GmbH Germany, part of Springer Nature 2022

\begin{abstract}
Metabolic pathways drive cellular behavior. Severe acute respiratory syndrome coronavirus 2 (SARS-CoV-2) infection causes lung tissue damage directly by targeting cells or indirectly by producing inflammatory cytokines. However, whether functional alterations are related to metabolic changes in lung cells after SARS-CoV-2 infection remains unknown. Here, we analyzed the lung single-nucleus RNA-sequencing (snRNA-seq) data of several deceased COVID-19 patients and focused on changes in transcripts associated with cellular metabolism. We observed upregulated glycolysis and oxidative phosphorylation in alveolar type 2 progenitor cells, which may block alveolar epithelial differentiation and surfactant secretion. Elevated inositol phosphate metabolism in airway progenitor cells may promote neutrophil infiltration and damage the lung barrier. Further, multiple metabolic alterations in the airway goblet cells are associated with impaired muco-ciliary clearance. Increased glycolysis, oxidative phosphorylation, and inositol phosphate metabolism not only enhance macrophage activation but also contribute to SARS-CoV-2 induced lung injury. The cytotoxicity of natural killer cells and CD8 ${ }^{+} \mathrm{T}$ cells may be enhanced by glycerolipid and inositol phosphate metabolism. Glycolytic activation in fibroblasts is related to myofibroblast differentiation and fibrogenesis. Glycolysis, oxidative phosphorylation, and glutathione metabolism may also boost the aging, apoptosis and proliferation of vascular smooth muscle cells, resulting in pulmonary arterial hypertension. In conclusion, this preliminary study revealed a possible cellular metabolic basis for the altered innate immunity, adaptive immunity, and niche cell function in the lung after SARS-CoV-2 infection. Therefore, patients with COVID-19 may benefit from therapeutic strategies targeting cellular metabolism in future.
\end{abstract}

Keywords COVID-19 $\cdot$ Cellular metabolism $\cdot$ Glycolysis $\cdot$ Host immunity $\cdot$ RNA sequencing

Edited by Deyin Guo.

Shuangyan Li, Fuxiaonan Zhao, Jing Ye and Kuan Li contributed equally to this work.

\author{
Qi Wu \\ wq572004@163.com \\ $\triangle$ Huaiyong Chen \\ huaiyong.chen@foxmail.com \\ 1 Department of Basic Medicine, Haihe Clinical School, \\ Tianjin Medical University, 890 Jingu Road, Tianjin 300350, \\ China \\ 2 Department of Basic Medicine, Haihe Hospital, Tianjin \\ University, 890 Jingu Road, Tianjin 300350, China
}

3 Key Research Laboratory for Infectious Disease Prevention for State Administration of Traditional Chinese Medicine, Tianjin Institute of Respiratory Diseases, 890 Jingu Road, Tianjin 300350, China

4 Tianjin Key Laboratory of Lung Regenerative Medicine, Haihe Hospital, Tianjin University, 890 Jingu Road, Tianjin 300350, China 


\section{Introduction}

Coronavirus disease 2019 (COVID-19), caused by severe acute respiratory syndrome coronavirus 2 (SARS-CoV-2), has spread worldwide. More than 273 million cases have been confirmed, and the death toll has exceeded 5 million as of December 17, 2021 (https://www.worldometers.info/ coronavirus/). The phenotypic traits of infected patients vary widely, from asymptomatic, mild, and moderate clinical presentations (including fever, dry cough, fatigue, headache, nausea, among others) to critical illness, which may cause intensive care unit (ICU) admission, and eventually death [1]. As the primary target of SARS-CoV-2, lung tissue displays massive pathologies after infection, including acute bronchiolitis, pulmonary edema, alveolar septal thickening, diffuse alveolar damage, fibrosis, pulmonary hemorrhage, micro-thrombi, and pulmonary hypertension [2-5].

Generally, a host responds to infections by eliciting nonspecific innate and adaptive immune responses. Physical barriers are vital components of the innate immune response that can directly hinder pathogen invasion into the body. The epithelial barrier in the upper respiratory tract is essential for mucociliary clearance and prevents respiratory pathogens from entering the lower respiratory tract. Submucosal glands, mucous cells, and goblet cells are the major sources of mucins in the human airways. Goblet cells are daughter cells of progenitor club cells, which are generated by basal cells or variant club cells upon injury [6, 7]. Multiple epithelial cells express angiotensin-converting enzyme 2 (ACE2) and become targets of SARS-CoV-2, including basal cells, club cells, and alveolar type 1 (AT1) and AT2 cells $[8,9]$. After SARS-CoV-2 infection, monocyte-derived macrophages migrate to the lungs to phagocytose infected cells and cellular debris [10].

The severity of COVID-19 appears to be related to the polarization of macrophages [11]. Neutrophils are recruited into the lungs to eliminate the virus by forming neutrophil extracellular traps and aggregates of histones and proteases [11]. However, the antigen presentation capacity of neutrophils may be reduced in patients with COVID-19 [12, 13]. Dendritic cells (DCs) produce antiviral cytokines, including type I interferons, and present processed viral antigens to $\mathrm{T}$ cells. NK cells, which are cytotoxic to virus-infected cells, can also recognize invading viruses. NK cells limit tissue damage by suppressing the inflammatory response [13]. The adaptive immune response is slower during initial pathogen infection but is more specific and effective than the innate immune response. In addition, the adaptive immune response can form immune memory after the initial pathogen infection, so the body can respond quickly to eliminate the same pathogen as the first infection. In response to SARS-CoV-2, $\mathrm{B}$ cells differentiate into plasma cells capable of secreting neutralizing antibodies [14]. Cytotoxic $\mathrm{CD} 8^{+} \mathrm{T}$ cells directly kill infected cells and secrete antiviral cytokines. $\mathrm{CD}^{+} \mathrm{T}$ cells coordinate and optimize the humoral response to viral infection [15]. Together with structural cells, cellular components in the innate and adaptive immune responses collaborate to restore lung homeostasis.

Changes in cellular metabolism during lung injury affect cell behavior, including survival, growth, and differentiation. We previously demonstrated that AT2 cells had upregulated glycolysis but downregulated fatty acid synthesis during bleomycin-induced alveolar epithelial injury [16]. The metabolic switch from glycolysis to lipid metabolism worsens bleomycin-induced alveolar epithelial injury [16]. Glucose deprivation or glycolysis inhibition promotes the differentiation of airway epithelial progenitor cells into ciliated and goblet cells [17]. Immuno-metabolic reprogramming may also occur in innate and adaptive immune cells after SARS-CoV-2 infection and contribute to the disease progression of COVID-19 [18]. Elevated glycolytic flux contributes to SARS-CoV-2 replication in monocytes and eventually induces pro-inflammatory cytokine production [19]. In IL-2/IL-12-stimulated NK cells, glutamine-dependent cMyc can regulate NK cell glycolysis and oxidative phosphorylation (OXPHOS), resulting in a robust NK cell response [20]. Myc-dependent metabolic reprogramming can regulate $\mathrm{CD}^{+} \mathrm{T}$ cell fate and is associated with memory lymphocyte generation [21]. A metabolic shift from oxidative phosphorylation to glycolysis may occur after SARS-CoV-2 infection as pyruvate kinase isozyme, hexokinase 2, and cytosolic lactate levels were all upregulated in COVID-19 [22, 23]. Such shift in immune cells can lead to hyper-inflammation and a cytokine storm, which result in exacerbated tissue damage [22]. Hu et al. observed a relationship between reduced serum HDL-cholesterol and the disease severity of the virus infection [24]. They also found that HDL-cholesterol concentration has positive correlation with $\mathrm{C}$-reactive protein (CRP), and negative correlation with lymphocytes count [24]. Furthermore, it has been also proposed that lipid-lowering therapeutic strategies, including statins and lipoprotein apheresis, may serve as strategies to reduce clinical complications in critical COVID-19 patients [25]. SARS-CoV-2 infection-induced changes in amino acid metabolism connected with disease severity and lung damage [26]. Revealing the mechanisms of metabolic reprogramming in different host cells will help us understand SARS-CoV-2-induced lung immuno-pathogenesis.

Here, by reanalyzing single-nucleus RNA-sequencing (snRNA-seq) data obtained from the lungs of deceased COVID-19 patients, we aimed to reveal the metabolic alterations in cellular components of the innate immune system, adaptive immune system, and structural niche after SARS-CoV-2 infection. We found that changes in several 
metabolic pathways, such as glycolysis, OXPHOS, fatty acid metabolism, glutathione metabolism, inositol phosphate metabolism, may be associated with impaired barrier protection, immunity dysfunction, and pulmonary fibrosis and arterial hypertension, which could facilitate COVID19 progression or even death.

\section{Methods}

\section{Data collection}

The single-nucleus RNA-sequencing (snRNA-seq) data of lung tissues from 19 deceased COVID-19 patients (12 men and 7 women) and 7 controls (4 men and 3 women) were obtained from the GEO database (accession number: GSE171524). In the COVID-19 cohort, the median postmortem interval was $4 \mathrm{~h}$, and the median age was 72 years. Control individuals with a median age of 70 years underwent lung biopsy/resection before the SARS-CoV-2 epidemic. The metadata information and cell type identification of all human cells in this study were referred to the original report [27].

\section{Score metabolism-related KEGG pathways}

The metabolism-related Kyoto Encyclopedia of Genes and Genomes (KEGG) terms were downloaded from the KEGG database (https://www.kegg.jp/), and a total of 35 metabolic pathways, and 12 metabolism-associated signaling pathways were used for further analysis. The AddModuleScore function in Seurat was used to calculate the score of each KEGG pathway in each cell. Data visualization was performed using the "ggplot2" R package (3.3.5), and only KEGG pathways with $P$ value $<0.01$ were displayed. Red dots indicate upregulated metabolic pathways in COVID-19 patients, and blue dots indicate downregulated metabolic pathways. A darker color indicates a smaller $P$ value.

\section{Score lung cell function}

The AddModuleScore function in the Seurat package was used to calculate the functional signatures of each cell cluster. The aging score was calculated using genes in the GO term aging (GO: 0,007,568). The apoptosis score was calculated using the genes in the apoptosis pathway (hsa04210). The club host defense score was calculated using SCGB1A1, CXCL17, CXCL1, CXCL6, C3, CD55,
B2M, PIGR, TLR5, LCN2, LYZ, BPIFB1, GSTP1, RDH10, AKR1C1, DHRS9, CYP2F1, MGST1, CYP4B1, SERPINA1, SLPI, WFDC2, SERPINB3, CST3, CTNNB 1, CXADR, CLDN1, CLDN4, OCLN, TFF3, MUC1, MUC5B, ITGAV, and ITGBI [28]. The mucus secretion score was calculated using genes in the GO term mucus secretion (GO: $0,070,254)$. The surfactant hemostasis score was calculated using the genes in the GO term surfactant hemostasis (GO: $0,043,129)$. The macrophage activation score was calculated using genes in the GO term macrophage activation (GO: $0,042,116)$. The macrophage chemotaxis score was calculated using genes in the GO term macrophage chemotaxis (GO: $0,048,246)$. The macrophage proliferation score was calculated using genes in the GO term macrophage proliferation (GO: 0,061,517).

The NK activation score was calculated using genes in the GO term natural killer cell activation (GO: 0,030,101). The NK chemotaxis score was calculated using genes in the GO term natural killer cell chemotaxis (GO: $0,035,747)$. The NK degranulation score was calculated using genes in the GO term natural killer cell degranulation (GO: 0,043,320). The NK cell proliferation score was calculated using genes in the GO term natural killer cell proliferation (GO: 0,001,787). The DC chemotaxis score was calculated using genes in the GO term dendritic cell chemotaxis (GO: $0,002,407)$. The DC differentiation score was calculated using genes in the GO term dendritic cell differentiation (GO: 0,097,028). The T cell activation score was calculated using genes in the GO term $\mathrm{T}$ cell activation (GO: 0,042,110). The $\mathrm{T}$ cell chemotaxis score was calculated using genes in the GO term $\mathrm{T}$ cell chemotaxis (GO: $0,010,818$ ). The $\mathrm{T}$ cell cytotoxicity score was calculated using genes in the GO term T cell mediated cytotoxicity (GO: $0,001,913)$. The $\mathrm{T}$ cell proliferation score was calculated using genes in the GO term $\mathrm{T}$ cell proliferation (GO: 0,042,098). The B cell activation score was calculated using genes in the GO term $B$ cell activation (GO: 0,042,113). The B cell chemotaxis score was calculated using genes in the GO term B cell chemotaxis (GO: $0,035,754)$. The fibroblast proliferation score was calculated using genes in the GO term fibroblast proliferation (GO: 0,048,144). The fibroblast migration score was calculated using genes in the GO term regulation of fibroblast migration (GO: 0,010,762). The vascular smooth muscle cell migration score was calculated using genes in the GO term vascular associated smooth muscle cell migration (GO:1,904,738). The vascular smooth muscle cell proliferation score was calculated using genes in the GO term regulation of vascular associated smooth muscle cell proliferation (GO:1,904,705). 


\section{Correlation analysis between metabolism-related KEGG pathways and cellular functions}

The corr.test function in the psych (v2.1.9) package was used to evaluate the correlation between KEGG pathways and cellular functions based on the scores calculated above. Only correlations with $P$ value $<0.05$ and Pearson correlation $>0.2$ or $<-0.2$ were displayed. Red dots indicate positive associations between pathways and cellular functions, while blue dots represent negative associations. Darker colors indicate stronger correlations.

\section{Differential expression gene identification and visualization}

The Seurat function "FindMarkers" (version 4.0.2) was used for differential expression gene (DEG) analysis between COVID-19 patients and controls. Genes with a fold change (FC) greater than 1.2 or less than 0.83 and an adjusted $P<0.05$ were regarded as significantly differentially expressed genes. Significance was evaluated using the Wilcoxon rank-sum test. We analyzed the differentially expressed genes of specific metabolic pathways in certain cell types between the two groups. The genes included in specific metabolic pathways were determined using the Kyoto Encyclopedia of Genes and Genomes (KEGG) database. Data visualization was performed using R 4.1.0.

Circular visualization plots were produced using the "circlize" R package (version 0.4.13). Bars on the outside ring of each cell type represent the fold changes of DEGs as indicated between the COVID-19 and control groups. Red bars represent DEGs in each metabolic pathway with FC greater than 1.41 or less than 0.71 . The blue bars inside the ring indicate adjusted $P$-values that were statistically significant $(<0.05)$ between the COVID-19 and control groups.

The FC visualization of genes included in certain pathways was performed using the "pheatmap" R package (version 1.0.12). The heatmap shows DEGs (adjusted $P$-values less than 0.05) with an FC greater than 1.2 or less than 0.83. Red squares indicate the downregulated genes, and the green squares indicate the upregulated genes. The numbers in the squares represent the fold changes of the indicated genes between COVID-19 and control in each cell type. The violin plots were produced using the "ggplot2" R package (3.3.5).

\section{Results}

\section{Metabolic changes in each cell cluster in COVID-19 patients}

The clearance of SARS-CoV-2 from its major target organ depends on the potency of the innate immune system and adaptive immune system in the lung. The functional performance of immune cells and lung epithelial cells in these systems is tightly controlled by cellular metabolism. To reveal the cellular metabolic basis of SARS-CoV-2-induced death, we retrieved the snRNA-seq transcriptome of 19 deceased COVID-19 patients and 7 control subjects from publicly available datasets of the Gene Expression Omnibus (GSE171524). We first assessed and mapped the metabolic landscape of all cell types, and then analyzed several functions of a certain cell type to reveal functional alterations after SARS-CoV-2 infection. Next, we performed a correlation analysis between metabolic pathways and cellular functions, disclosing the potential associations between altered metabolic pathways and cellular functions. Furthermore, we focused on the DEGs associated with 19 metabolic pathways in 41 cell types and attempted to identify altered genes involved in a certain metabolic pathway that may influence host defense against SARS-CoV-2 infection (Fig. 1, Figure S1-S5).

A total of 35 metabolic pathways and 12 metabolismrelated signaling pathways were analyzed. Most metabolic pathways and metabolism-related signaling pathways were upregulated in COVID-19 patients as compared to the control group (Fig. 2). Macrophage, lung fibroblast and epithelial cells exhibited more upregulated metabolic pathways among 41 cell types investigated. While goblet cells, ciliated cells, alveolar fibroblasts, vascular smooth cells, plasma cells and neuronal cells have more downregulated metabolic pathways than other cell types. Most of these cells express ACE2, so SARS-CoV-2 invasion may perturb the transcription activity, causing metabolic alterations in the infected cells. Other mechanisms, such as hypoxia or hyper-inflammation in COVID-19, may also influence transcription activity and cellular metabolism in these cells.

Similarities within one hierarchy of cells was observed in terms of SARS-CoV-2 infection-induced cellular metabolic changes. Airway epithelial cells, including basal cells, club cells, ciliated cells, and airway mucous cells, exhibited upregulated OXPHOS (Fig. 2). In addition to OXPHOS, alveolar epithelial cells including alveolar type 1 (AT1) cells and alveolar type 2 (AT2) cells upregulated many other metabolic pathways, for example glycolysis, fatty acid biosynthesis, degradation and elongation, glycerolipid metabolism, glycerophospholipid metabolism, purine and pyrimidine metabolism, and cysteine and methionine metabolism (Fig. 2). For most immune cells, biosynthesis of unsaturated fatty acids, fatty acid elongation, and glycolysis/gluconeogenesis pathways were commonly upregulated. OXPHOS, glycolysis/gluconeogenesis, glutathione metabolism and arachidonic acid metabolism represented the most upregulated metabolic pathways in smooth muscle cells. Glycolysis/gluconeogenesis, OXPHOS, glutathione metabolism, fatty acid 


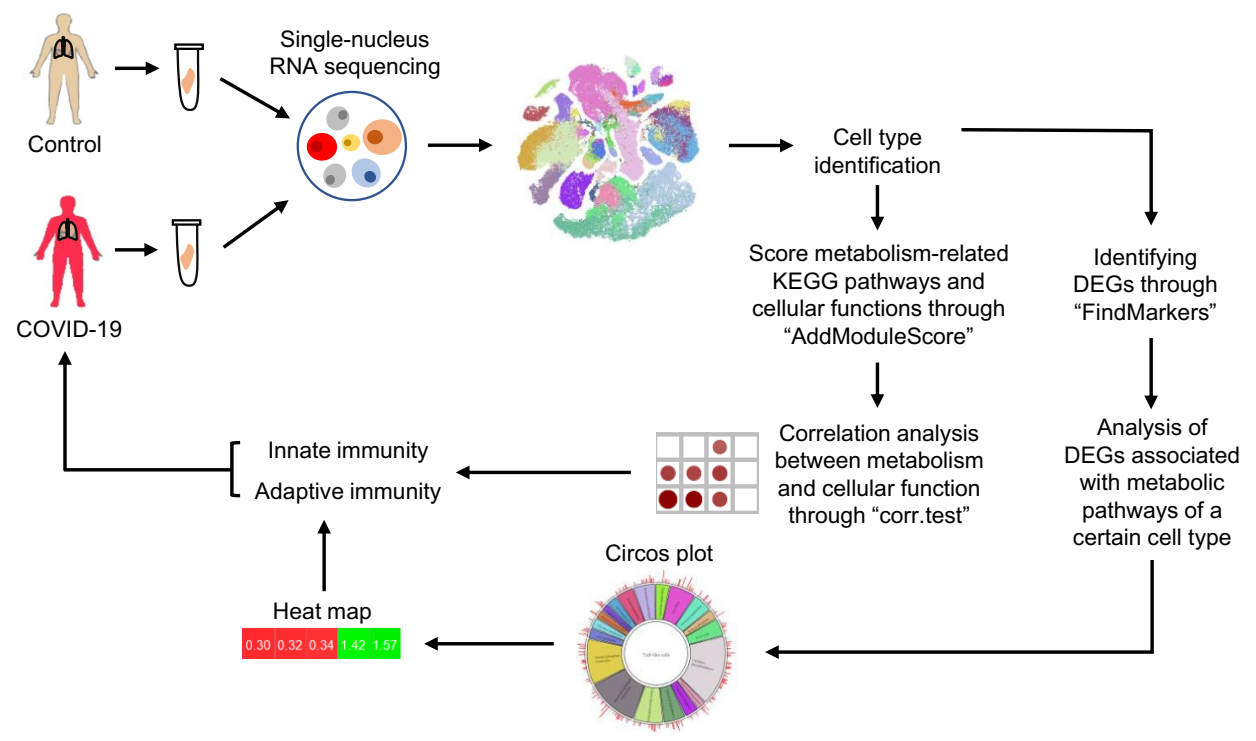

Fig. 1 Simplified outline for snRNA-seq data analysis. snRNA-seq data from COVID-19 and control lung tissue were downloaded from the GEO database. "AddModuleScore" was used to score the metabolism-related KEGG pathways and Gene Ontology Terms associated with cellular functions. Then corr.test function was used to perform correlation analysis between metabolism-related pathways and cel- lular functions. "FindMarkers" was used to identify the Differential Expression Genes (DEGs) between the two groups. A circos plot and a heat map were used to visualize the DEGs associated with metabolic pathways of certain cell types to reveal the metabolic alterations relevant to innate or adaptive immunity

pathways. Pyruvate kinase ( $P K M)$ catalyzes the transfer of phosphoenolpyruvate to pyruvate, the last step of glycolysis. Upon glucose deprivation, acyl-CoA synthetase short-chain family 2 (ACSS2) generates acetyl-CoA for histone acetylation. Both PKM and ACSS2 transcripts were upregulated in club cells during COVID-19 (Fig. 3c). Previous findings suggested that altered glucose metabolism is associated with enhanced chemotaxis of monocytes and neutrophils into the lungs, resulting in a poor prognosis in critically ill patients [30]. Acyl-CoA synthetase long-chain family member 1 (ACSL1), a key rate-limiting enzyme in fatty acid oxidation, was also elevated at the transcriptional level in club cells after SARS-CoV-2 infection (Fig. 3c). Inositol phosphates (InsPs) are important second messengers that participate in signal transduction and regulate various biological functions. Phospholipase $\mathrm{C}(P L C)$ hydrolyzes phosphatidylinositol-4,5-bisphosphate (PIP2) to generate inositol-1,4,5triphosphate (IP3) and diacylglycerol (DAG) [31]. We found enhanced expression of PLC-gamma-2 (PLCG2) transcripts in club cells after SARS-CoV-2 infection (Fig. 3c). A similar increase was also found in the expression of phosphatidylinositol-4,5-bisphosphate 3-kinase catalytic subunit alpha (PIK3CA) (Fig. 3c), which can catalyze the phosphorylation of phosphatidylinositol-4,5 bisphosphates (PIP2) to phosphatidylinositol-(3,4,5)-triphosphate (PIP3).

Goblet cells are daughters of club cells found in the lungs. Goblet cell hyperplasia is a hallmark of bronchial asthma, cystic fibrosis, and chronic obstructive pulmonary

enhanced expression levels of several genes involved in these 


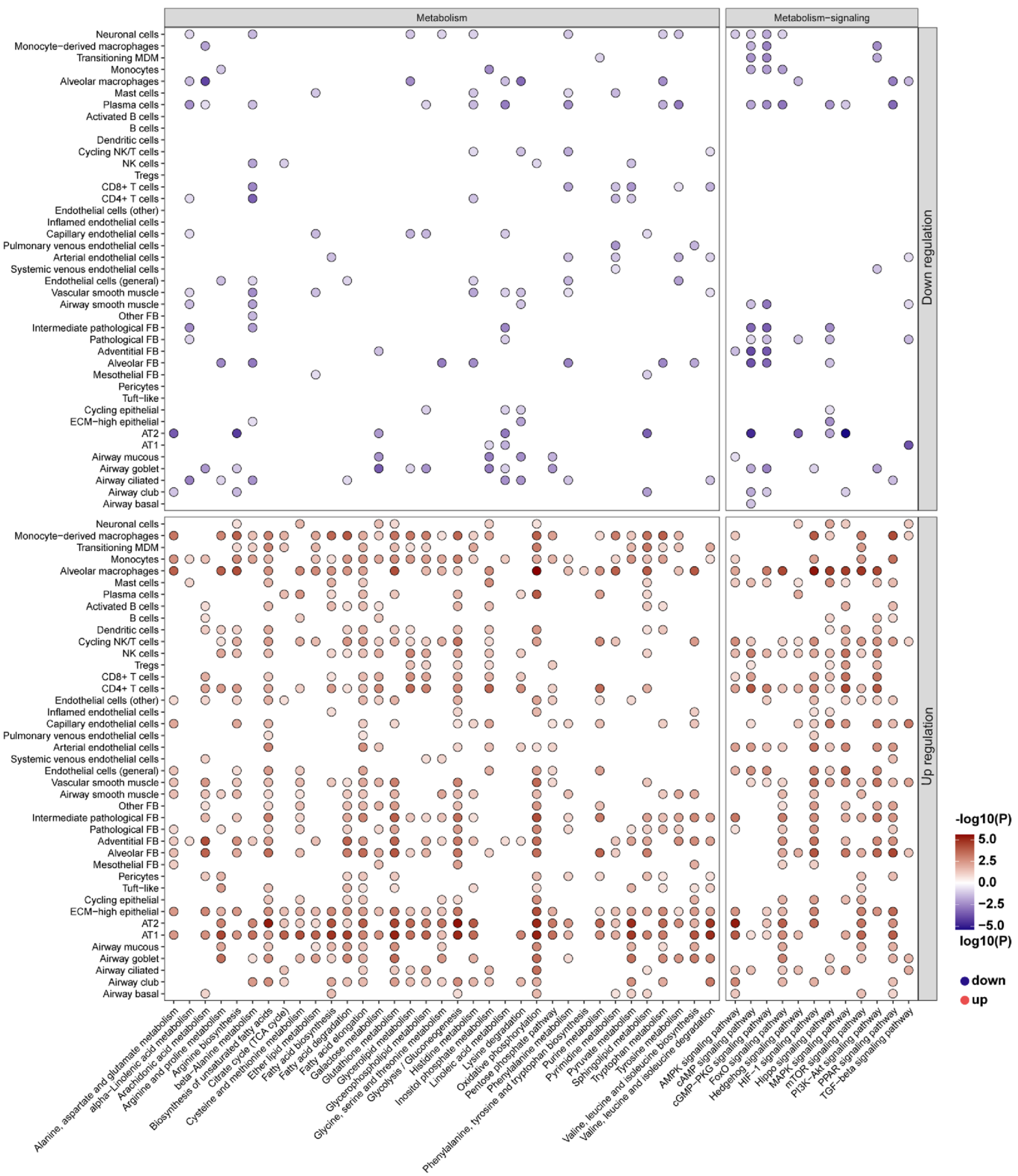

Fig. 2 The significantly altered metabolic pathways (left panel) and metabolism-related signaling pathways (right panel) in each cell cluster in COVID-19 patients (Student's $t$-test; $P$ value $<0.01$ ). Red dots indicate the upregulated metabolic pathways in COVID-19 patients and blue dots indicate the downregulated metabolic pathways. Darker colors indicate a smaller $P$ value 
diseases. We found that aging and apoptosis were slightly upregulated, while mucus secretion was significantly downregulated in COVID-19 patients compared to control group (Fig. 3d). Correlation analysis suggested that OXPHOS, glutathione metabolism and FoxO signaling pathway were positively associated with both aging and apoptosis in goblet cells (Fig. 3e). Besides, aging was also positively correlated with valine, leucine and isoleucine biosynthesis in goblet cells (Fig. 3e). Compared to club cells, airway goblet cells displayed more DEGs involved in metabolic pathways, manifested by more red bars in the outer ring and more blue bars in the inner ring in the circos plot (Fig. 3f). Multiple genes in OXPHOS were upregulated, while the expression levels of multiple genes in inositol phosphate metabolism decreased (Fig. 3g), consistent with above metabolism score in goblet cells (Fig. 2). Glycolysis, lipid metabolism, and mitochondrial OXPHOS pathways are associated with goblet cell differentiation and mucus secretion in goblet cells [32]. Expression levels of multiple types of mucins were decreased, including MUC5AC, MUC3A and MUC16 (Fig. 3h), in accordance with above function score (Fig. 3d). Adenosine triphosphate (ATP)-induced mucin secretion correlates with PLC activation and downstream activation of protein kinase $\mathrm{C}$ (PKC) via diacylglycerol [33]. SARS-CoV-2 infection resulted in a decreased expression of $P L C B 1, P L C E 1$, and $P L C H 1$ and an increased expression of PLCG2 (Fig. 3g). Aberrant mucin secretion may explain the impaired mucociliary clearance in COVID-19 [34].

\section{Cellular metabolic changes illustrating impaired alveolar repair and surfactant transportation in COVID-19}

Two major cell types in the alveolar gas exchange region, AT2 and AT1 cells, exhibited massive metabolic alterations after SARS-CoV-2 infection. We found that aging was significantly upregulated in AT2 cells, which is consistent with other findings [35]. Surfactant hemostasis was markedly downregulated in COVID-19 patients (Fig. 4a). Correlation analysis showed that OXPHOS and glutathione metabolism were positively associated with aging in AT2 cells. In addition, MAPK and cAMP signaling pathways may be positively correlated with apoptosis in AT2 cells (Fig. 4b). Glycolysis, oxidative phosphorylation, pyruvate metabolism, fatty acid metabolism, histidine metabolism, and glycerolipid metabolism were enhanced in AT2 cells (Figs. 2, $4 c)$. Consistent with the metabolism scoring above, the expression levels of most DEGs related to glycolysis or fatty acid metabolism were elevated (Fig. 4d). We observed that some isozymes were upregulated but others downregulated in fatty acid oxidation pathway, reflecting changes in the utilization of substrates in AT2 cells after SARS-CoV-2 infection. Enhanced glycolytic metabolism may hinder AT1 cell differentiation during IL-1 $\beta$-mediated inflammation [36]; therefore, altered glucose metabolism may impair the differentiation of AT2 cells into mature AT1 cells.

Surfactants produced by AT2 cells decrease the surface tension necessary for gas exchange and the structural integrity of alveoli [37]. Surfactants mainly consist of phospholipids, such as phosphatidylcholine (PC), phosphatidylglycerol (PG), and other surfactant proteins (SPs), including SP-A, SP-B, SP-C, and SP-D. SP-A can bind microbial pathogens, such as viruses, fungi, and bacteria, exerting a critical role in innate host defense and anti-inflammation [37, 38]. The functional score showed impaired surfactant homeostasis after SARS-CoV-2 infection (Fig. 4a). We found an increased expression of SP-A1 and SP-A2 transcripts in AT2 cells in patients with COVID-19 (Fig. 4e). In addition, the expression of fatty acid synthase $(F A S N)$ was upregulated in COVID-19 (Fig. 4d). FASN catalyzes de novo fatty acid biosynthesis, which is an important precursor for pulmonary surfactant biosynthesis. While the gene expression level of surfactant transporting protein, ATP binding cassette subfamily A member 3 ( $A B C A 3$ ) (Fig. 4e), decreased. $A B C A 3$ is a member of $\mathrm{ABS}$ transporter family, which enriches in the lung and participates in surfactant synthesis, storage in lamellar bodies (LBs). Furthermore, mutations of this gene cause congenital surfactant deficiency [39]. Here we speculate that AT2 cells aim to increase expression of surfactants including both proteins and lipids to defense against pathogens, while abnormal surfactant transporting system may lead to surfactant deficiency finally. These data revealed the cellular metabolic basis of impaired alveolar epithelial regeneration and surfactant deficiency-induced defective defense against SARS-CoV-2.

Correlation analysis showed that multiple metabolic pathways or metabolism-related signaling pathways were positively correlated to aging or apoptosis of AT1 cells (Fig. 4f, g). Like AT2 cells, AT1 cells showed enhanced glycolysis, pyruvate metabolism, fatty acid metabolism, OXPHOS, glycerolipid metabolism, and glycerophospholipid metabolism after SARS-CoV-2 infection (Fig. 2). DEG analysis showed that many genes involved in these metabolic pathways were upregulated (Fig. 4h, i). The connection between the altered metabolism and gas exchange function of AT1 cells requires further research. Metabolic alterations that drive aging or apoptosis of AT1 and AT2 cells explained the occurrence of lung fibrotic progression in these COVID19 patients [27]. 
a

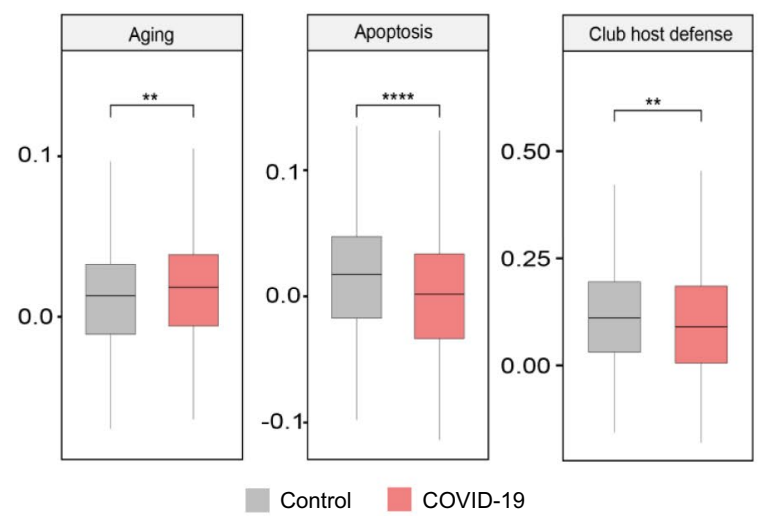

C

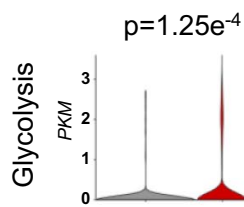

d
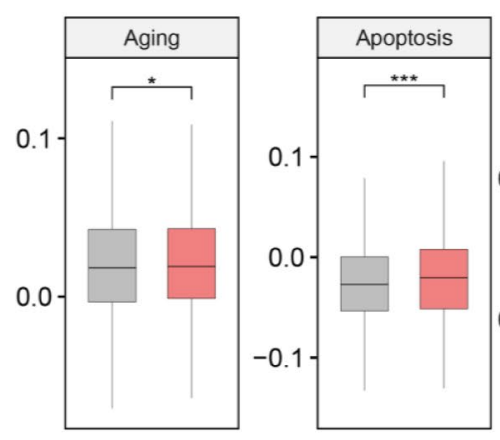

f
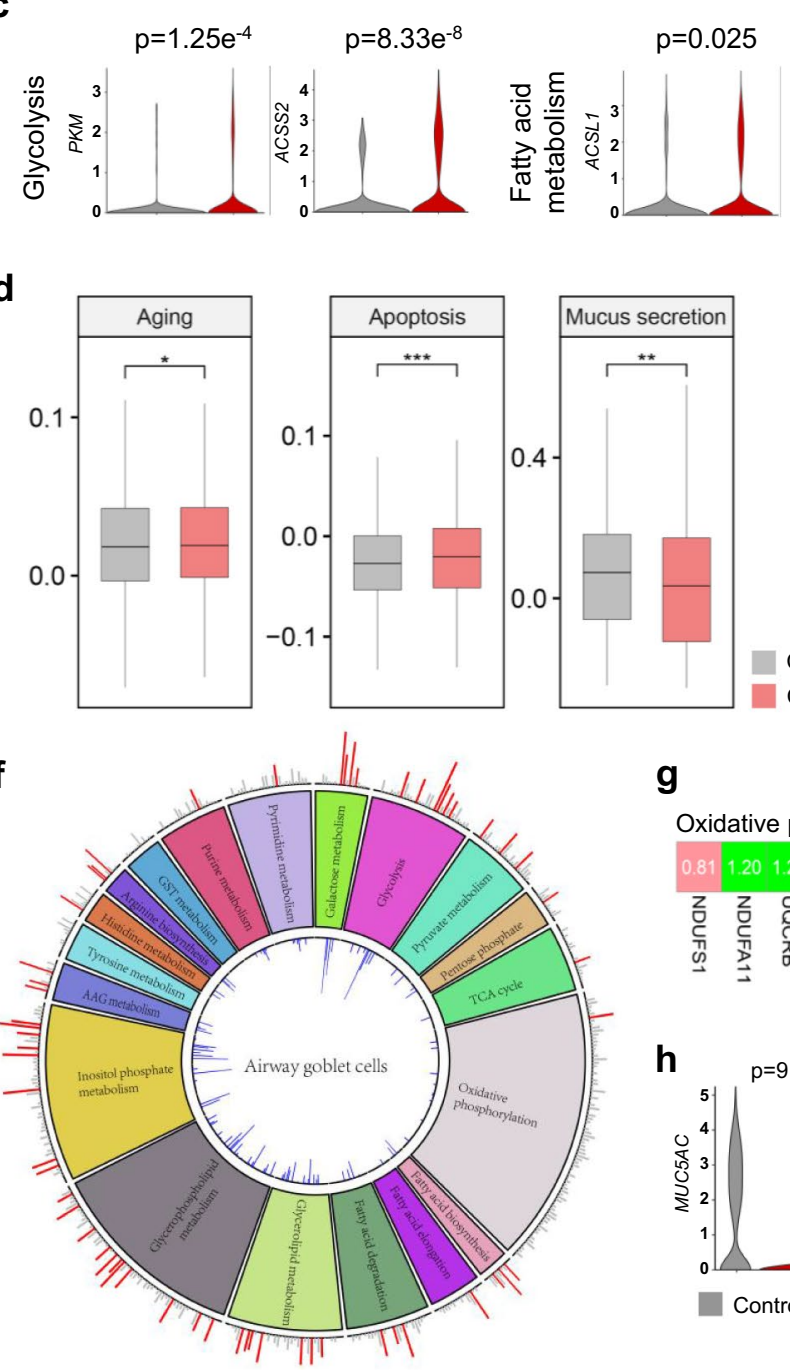

g

h b

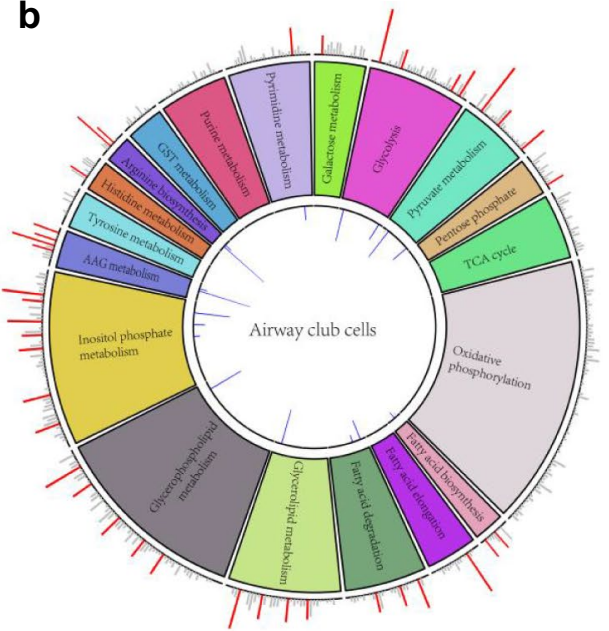

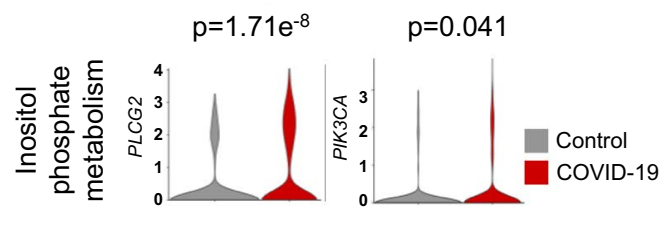

e

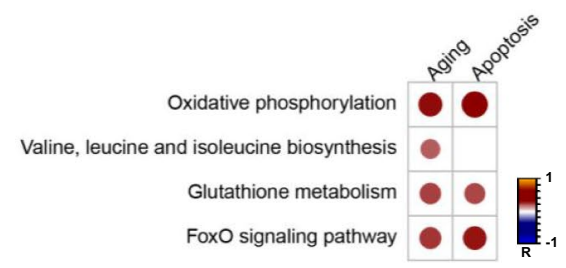

Oxidative phosphorylation Inositol phosphate metabolism

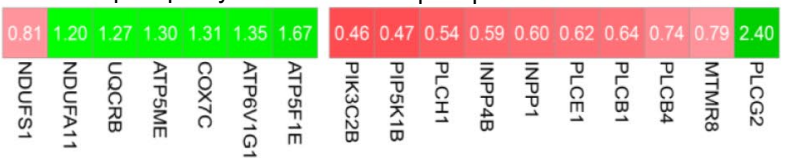
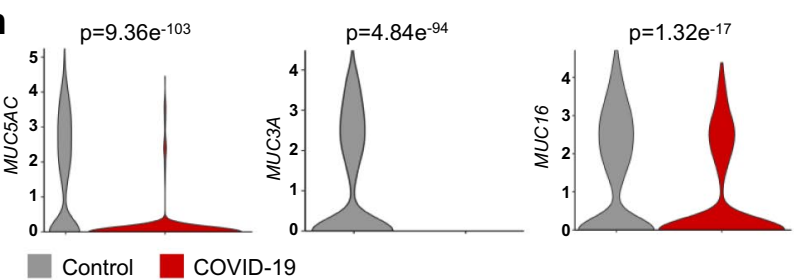

Control

COVID-19

Control COVID-19 
4Fig. 3 The metabolic processes that are altered in airway epithelial cells. a The selected functional alterations in airway club cells in COVID-19 patients (Student's $t$-test; $* * P<0.001$; $* * * * P<0.0001$ ). b Circos plots of airway club cells. The red bar on the outside indicates that the absolute value of $\log 2 \mathrm{FC}$ is greater than 0.5 . The blue bar suggests that the adjusted $P$ value is statistically significant $(<0.05)$. c Violin plots of PKM, ACSS2, ACSL1, PLCG2 and PIK3CA expression (log-normalized) in airway club cells from controls and patients with COVID-19. Wilcoxon rank-sum test with Bonferroni-adjusted $P$ value indicated on top. $\mathbf{d}$ The selected functional alterations in airway goblet cells in COVID-19 patients (Student's $t$-test; $* P<0.05$; $* * P<0.001 ; * * * P<0.001)$. e The Pearson correlations between the scores of goblet cell function and the metabolic pathways. " $R$ " indicates the Pearson correlation coefficient; only dots representing correlations with $|R|>0.2$ and $P$ value $<0.05$ are displayed. f Circos plots of airway goblet cells. The red bar on the outside indicates that the absolute value of $\log 2 \mathrm{FC}$ is greater than 0.5 . The blue bar suggests that the adjusted $P$ value is statistically significant $(<0.05)$. g Heat map demonstrating the fold changes of DEGs involved in a certain metabolic pathway in goblet cells. Green indicates the upregulated genes and red indicates the downregulated genes. The numbers in the squares represent the fold change of a certain gene, and darker colors indicate a larger fold change. h Violin plots of MUC5AC, MUC3A and MUC16 expression (log-normalized) in airway goblet cells from controls and patients with COVID-19. Wilcoxon rank-sum test with Bonferroni-adjusted $P$ value indicated on top. GST metabolism glycine, serine, threonine metabolism, AAG metabolism alanine, aspartate and glutamate metabolism

\section{SARS-CoV-2-induced metabolic reprogramming in innate immune cells}

Innate immunity against SARS-CoV-2 is built upon the first and rapid host defense immune cells, including neutrophils, eosinophils, basophils, NK cells, DCs, macrophages, and mast cells. Among these immune cells, macrophages exhibit remarkable metabolic changes (Fig. 2). Alveolar macrophages are the direct targets of SARS-CoV-2 through the S-protein-ACE2 interaction, resulting in their abnormal activation [40]. We observed that the scores of macrophage activation, chemotaxis, and proliferation were elevated in alveolar macrophages in COVID-19 patients (Fig. 5a). Macrophage activation and chemotaxis were positively correlated with OXPHOS, and macrophage activation positively correlated with the PI3K-Akt signaling pathway (Fig. 5b). Other altered metabolic pathways in alveolar macrophages, included glycolysis, pyruvate metabolism, fatty acid metabolism, inositol phosphate metabolism, glycerolipid metabolism, and glycerophospholipid metabolism (Figs. 2, 5c). It is worth mentioning that many elements of the OXPHOS pathway were increased at the transcript level in alveolar macrophages after SARS-CoV-2 infection (Fig. 5d). The expression levels of several genes coding for "vacuolar" ATPase (V-ATPase) were increased in COVID-19, including ATP6V0C, ATP6V1B2, ATP6V1C1, ATP6AP1, ATP6VOA1, ATP6VIA, ATP6VOB (Fig. 5d). These proteins are associated with phagosome acidification during the maturation process of phagosomes [41]. These data explained the activation and chemotaxis of alveolar macrophages in these COVID-19 patients [27]. Increased generation of intracellular IP3 and $\mathrm{Ca}^{+}$via activation of PLC promoted macrophage migration [42]. We observed higher expression levels of PLCG2 in the alveolar macrophages of COVID-19 patients compared to control subjects (Fig. 5d). In consistent with our findings, macrophages in the lungs of severe COVID-19 patients showed increased glycolysis, facilitating pro-inflammatory cytokine generation $[19,43]$. In the glycerolipid metabolism pathway, lipoprotein lipase (LPL) catalyzes the hydrolysis of triglycerides to free fatty acids (FFAs) and mediates lipoprotein uptake [44]. Very low-density lipoprotein (VLDL) uptake mediated by LPL was reported to inhibit macrophage inflammation through peroxisome proliferator-activated receptor- $\delta$ (PPAR $\delta$ ) activation $[45,46]$. There was a significant reduction in $L P L$ expression in the alveolar macrophages of patients with COVID-19 (Fig. 5d). Furthermore, inhibition of cellular glycolysis significantly reduced alveolar macrophage polarization to the M1 type [47]. The expression of $P K M$, along with several other glycolysis-related enzymes, such as phosphoglycerate kinase 1 $(P G K 1)$ and enolase 1 (ENOI), was upregulated in alveolar macrophages after SARS-CoV-2 infection (Fig. 5d). These data suggest that alveolar macrophages may present a proinflammatory phenotype rather than an anti-inflammatory phenotype, causing lung tissue damage.

SARS-CoV-2 infection blunts NK cell effector functions and hinders the effective clearance of infected cells [48]. The scores of NK cell activation, chemotaxis, degranulation, and proliferation were elevated in NK cells in these COVID-19 patients (Fig. 5e). Although the DEGs presented in the circos plot were not apparent, several metabolic pathways were significantly altered according to the metabolism score, including upregulated glycolysis, inositol phosphate metabolism, and glycerolipid metabolism (Figs. 2, 5f). The glycolytic rate of NK cells increases during viral infection and inflammation, and lactate dehydrogenase A ( $L D H A)$-deficient NK cells are unable to exhibit optimal antiviral activities [49]. $P L C G 2$-deficient NK cells fail to mobilize intracellular calcium and are unable to secrete cytotoxic granules, which are essential for killing virally infected cells [50]. There was an increase in PLCG2 expression in the COVID-19 group, likely boosting the cytotoxicity of NK cells (Fig. 5g). The expression levels of two enzymes in glycerolipid metabolism pathway, lipin 1 (LPIN1) and lipin 2 (LPIN2), were increased in NK cells after SARS-CoV-2 infection (Fig. 5g). These enzymes can dephosphorylate phosphatidic acid to yield DAG, which increases intracellular calcium levels and facilitates NK cell cytotoxicity. These metabolic changes may contribute to enhanced NK cell effector functions, such as chemotaxis, degranulation and cytotoxicity. 
a

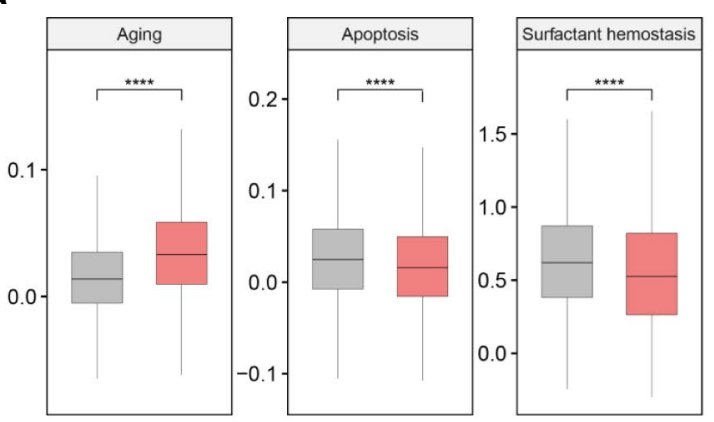

Control COVID-19 b

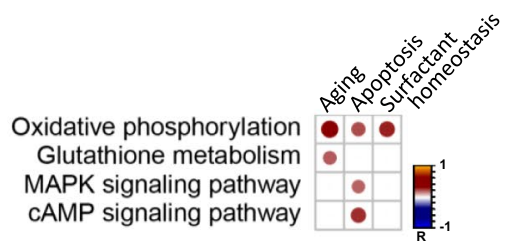

d Glycolysis

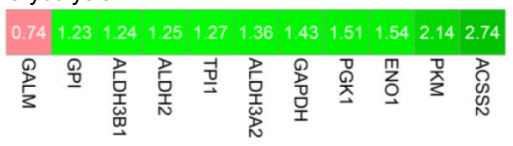

Fatty acid metabolism

$0.34 \quad 0.601 .201 .211 .22 \quad 1.22 \quad 1.24 \quad 1.251 .361 .401 .451 .64 \quad 2.40$

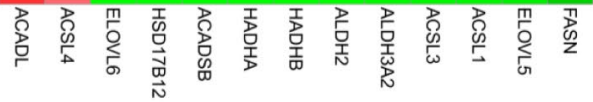

Glutathione metabolism

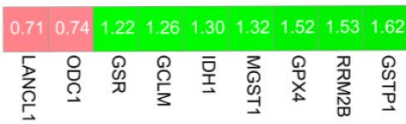
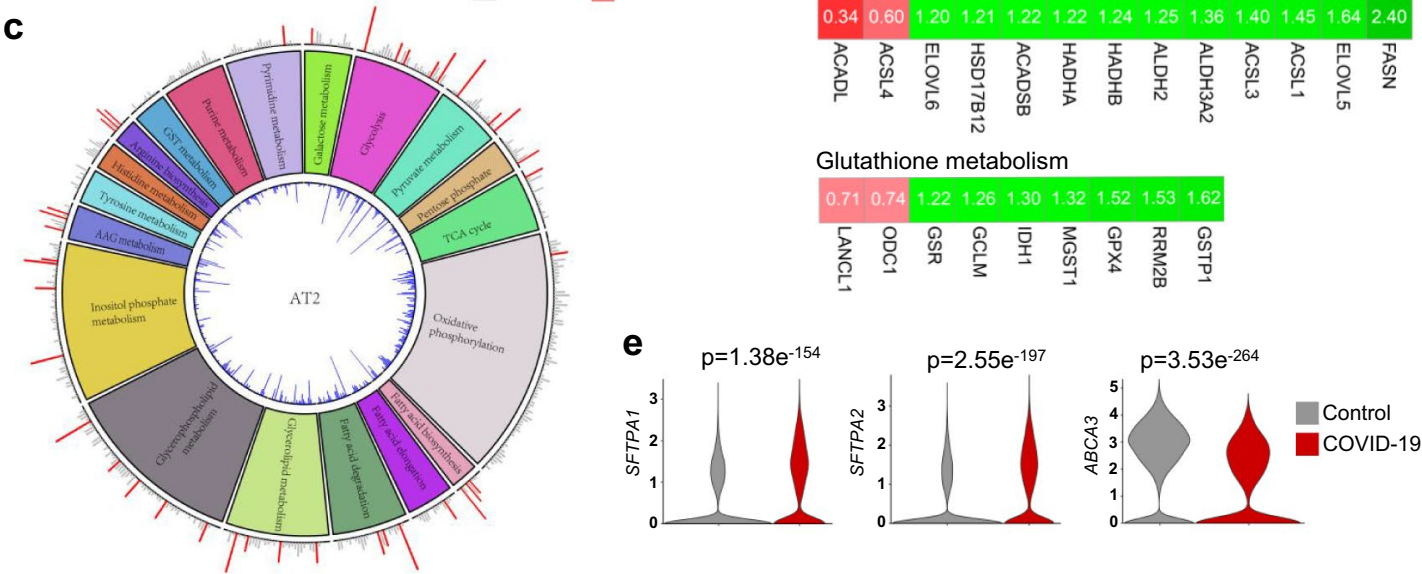

f

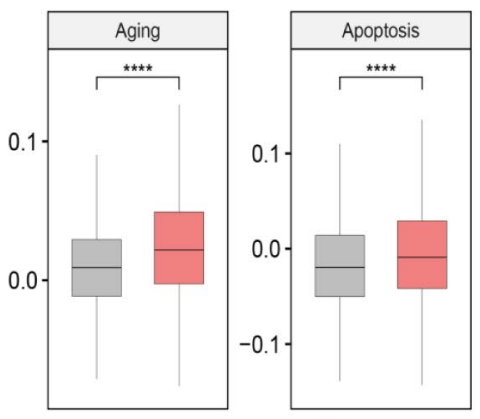

Control COVID-19

h

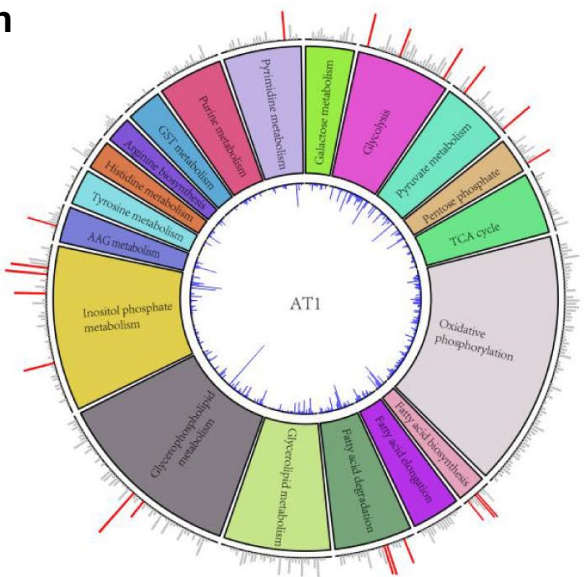

g

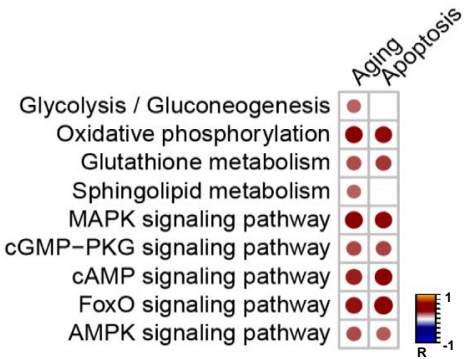

i

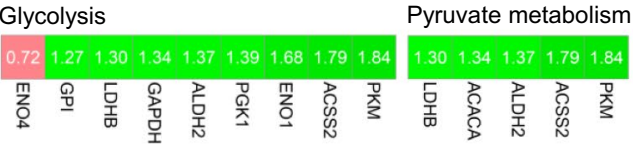

Fatty acid metabolism Glycerolipid metabolism 0.641 .201 .211 .341 .341 .371 .371 .531 .661 .710 .771 .251 .321 .371 .371 .40

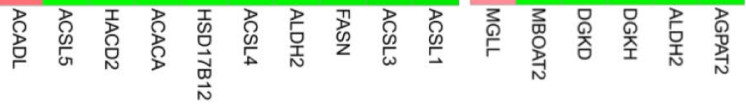

Oxidative phosphorylation

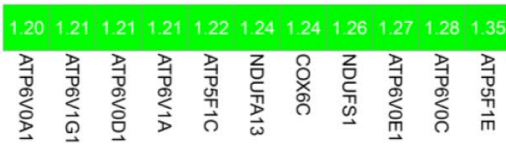

Glycerophospholipid metabolism

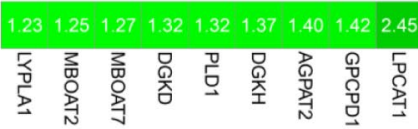


4Fig. 4 The metabolic pathways that are altered in alveolar type 1 (AT1) cells and alveolar type 2 (AT2) cells. a The selected functional alterations in AT2 cells in COVID-19 patients (Student's $t$-test; $* * * * P<0.0001)$. b The Pearson correlations between the scores of AT2 cell function and the metabolic pathways. " $R$ " indicates the Pearson correlation coefficient; only dots representing correlations with $|R|>0.2$ and $P$ value $<0.05$ are displayed. c Circos plots of AT2 cells. The red bar on the outside indicates that the absolute value of $\log 2 \mathrm{FC}$ is greater than 0.5 . The blue bar suggests that the adjusted $P$ value is statistically significant $(<0.05)$. d Heat map demonstrating the fold changes of DEGs involved in a certain metabolic pathway in AT2 cells. Green indicates the upregulated genes and red indicates the downregulated genes. The numbers in the squares represent the fold change of a certain gene, and darker colors indicate a larger fold change. e Violin plots of SFTPA1, SFTPA2 and ABCA3 expression (log-normalized) in AT2 cells from controls and patients with COVID-19. Wilcoxon rank-sum test with Bonferroni-adjusted $P$ value indicated on top. $\mathbf{f}$ The selected functional alterations in AT1 cells in COVID-19 patients (Student's $t$-test; $* * * * \mathrm{P}<0.0001$ ). $\mathbf{g}$ The Pearson correlations between the scores of AT1 cell function and the metabolic pathways. " $R$ " indicates the Pearson correlation coefficient; only dots representing correlations with $|R|>0.2$ and $P$ value $<0.05$ are displayed. $\mathbf{h}$ Circos plots of AT1 cells. The red bar on the outside indicates that the absolute value of $\log 2 \mathrm{FC}$ is greater than 0.5 . The blue bar suggests that the adjusted $P$ value is statistically significant $(<0.05)$. i Heat map demonstrating the fold changes of DEGs involved in a certain metabolic pathway in AT1 cells. Green indicates the upregulated genes and red indicates the downregulated genes. The numbers in the squares represent the fold change of a certain gene, and darker colors indicate a larger fold change. GST metabolism glycine, serine, threonine metabolism. AAG metabolism alanine, aspartate and glutamate metabolism

DCs are important antigen-presenting and interferonsecreting cells [51]. SARS-CoV-2 infection impairs the function of DCs during maturation, perhaps damaging the induction of adaptive $\mathrm{T}$ cell responses against virus infection [52]. DC chemotaxis was upregulated, while DC differentiation was downregulated in these COVID-19 patients (Fig. 5h). Like NK cells, the DEGs presented in the circos plot of DC cells were not very apparent, but several metabolic pathways were significantly upregulated according to the metabolism score, including glycolysis, OXPHOS, fatty acid elongation and degradation, inositol phosphate metabolism, and glycerolipid metabolism (Fig. 2, Fig. 5i). Several studies have implied that pyruvate kinase M2 (PKM2) is involved in DC functions [53,54]. We observed increased PKM expression in the COVID-19 group compared to the control group (Fig. 5j), likely contributing to DC activation, maturation, and antigen presentation.

\section{Changes in cell metabolism associated with adaptive immunity in COVID-19}

$\mathrm{CD}^{+} \mathrm{T}$ cells, $\mathrm{CD}^{+} \mathrm{T}$ cells, and plasma cells are the primary effector cells of the adaptive immune system. The normal functions of $\mathrm{CD} 4^{+} \mathrm{T}$ cells are necessary for $\mathrm{T}$-dependent $\mathrm{B}$ cells to secrete pathogen-specific antibodies. There were few metabolic changes in $\mathrm{CD} 4^{+} \mathrm{T}$ cells after SARS-CoV-2 infection (Figure S3). Cytotoxic T lymphocytes (CTLs), such as $\mathrm{CD}^{+} \mathrm{T}$ lymphocytes, are necessary for virus control and elimination. $\mathrm{T}$ cell activation, chemotaxis, cytotoxicity, and differentiation were all enhanced for $\mathrm{CD}^{+} \mathrm{T}$ cells in these COVID-19 patients (Fig. 6a). Despite the inconspicuous DEGs presented in the circos plot (Fig. 6b), multiple metabolic pathways were significantly upregulated in COVID-19 patients based on the metabolism score, including glycolysis, fatty acid metabolism, glycerolipid metabolism, and inositol phosphate metabolism (Fig. 2). Activation of T cell receptor (TCR) signaling can induce PLCG1 recruitment and activation, followed by elevated intracellular IP3 and DAG levels [55, 56]. Both PLCG1 and PLCG2 in the inositol phosphate metabolism pathway presented higher expression levels in $\mathrm{CD}^{+} \mathrm{T}$ cells in COVID-19 (Fig. 6c), likely promoting $\mathrm{T}$ cell activation and effective antiviral immune response. Besides LPC-induced DAG production, in the glycerolipid metabolism pathway, we found elevated expression of both LPIN1 and LPIN2 in CD8 ${ }^{+} \mathrm{T}$ cells after SARS-CoV-2 infection (Fig. 6c). DGK $\alpha$ and DGK $\zeta$ could inhibit $\mathrm{CD} 8^{+} \mathrm{T}$ cell primary antiviral immune activity by repressing their proliferation and cytokine production [57]. The SARS-CoV-2-induced increase in DGKH expression may limit the DAG-induced antiviral immune response as a negative feedback mechanism (Fig. 6c). 


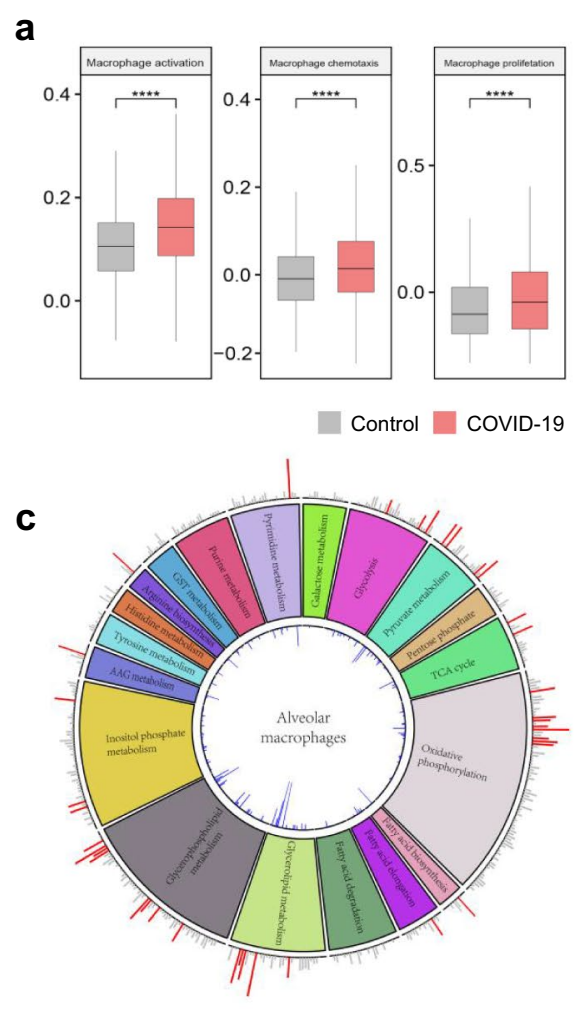

b

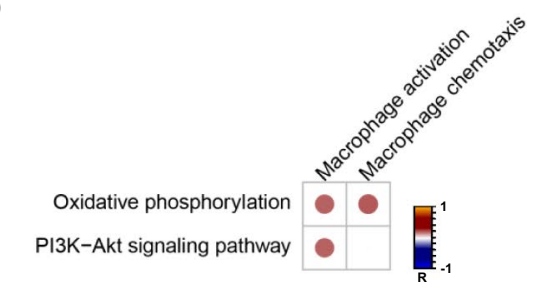

d Oxidative phosphorylation

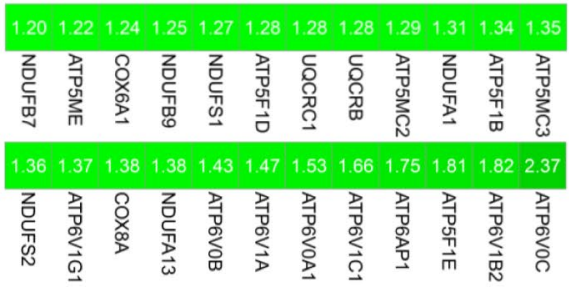

Inositol phosphate metabolism Glycolysis

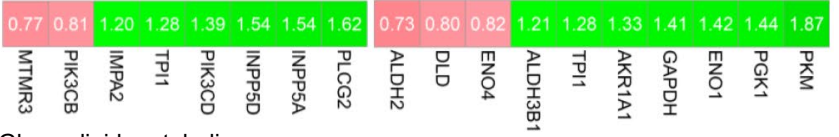

Glycerolipid metabolism

$\begin{array}{llllllllllllllllll}0.35 & 0.49 & 0.68 & 0.72 & 0.73 & 0.75 & 0.77 & 0.81 & 0.82 & 1.29 & 1.33 & 1.36 & 1.40 & 1.63 & 2.31\end{array}$

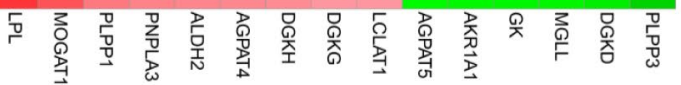

e

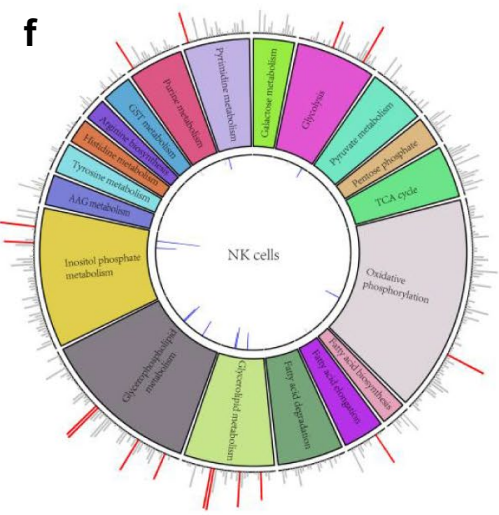

g Control $\square$ CoviD-19

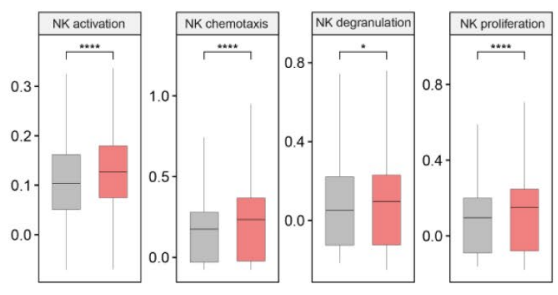

Control COVID-19

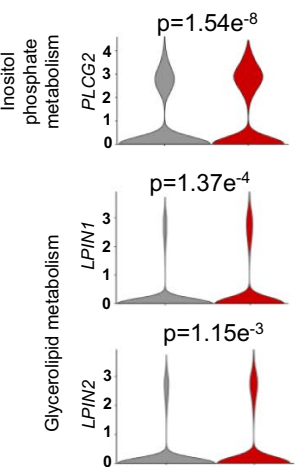

h

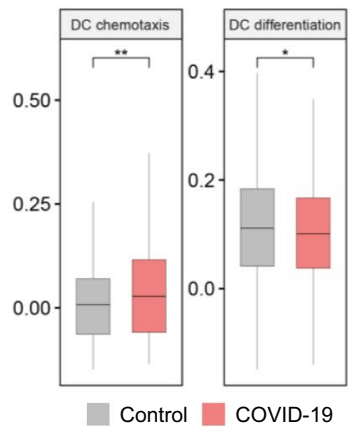

i

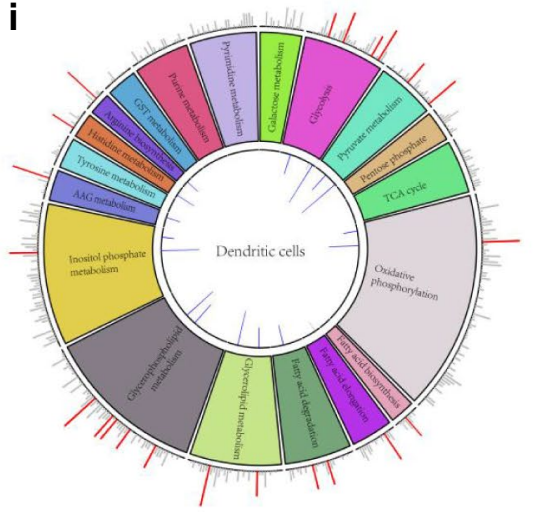

j

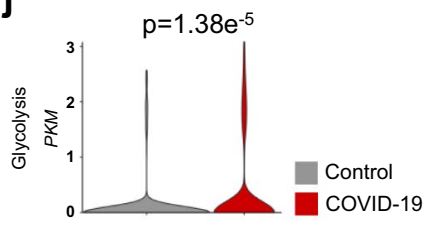


4Fig. 5 The metabolic pathways that are altered in alveolar macrophages, NK cells and DCs. a The selected functional alterations in alveolar macrophages in COVID-19 patients (Student's $t$-test; $* * * * P<0.0001)$. b The Pearson correlations between the scores of alveolar macrophages function and the metabolic pathways. " $R$ " indicates the Pearson correlation coefficient; only dots representing correlations with $|R|>0.2$ and $P$ value $<0.05$ are displayed. c Circos plots of alveolar macrophages. The red bar on the outside indicates that the absolute value of $\log 2 \mathrm{FC}$ is greater than 0.5 . The blue bar suggests that the adjusted $P$ value is statistically significant $(<0.05)$. d Heat map demonstrating the fold changes of DEGs involved in a certain metabolic pathway in alveolar macrophages. Green indicates the upregulated genes and red indicates the downregulated genes. The numbers in the squares represent the fold change of a certain gene, and darker colors indicate a larger fold change. e The selected functional alterations in NK cells in COVID-19 patients (Student's $t$-test; $* P<0.05 ; * * * * P<0.0001)$. f Circos plots of NK cells. The red bar on the outside indicates that the absolute value of $\log 2 \mathrm{FC}$ is greater than 0.5 . The blue bar suggests that the adjusted $P$ value is statistically significant $(<0.05)$. g Violin plots of PLCG2, LPIN1 and LPIN2 expression (log-normalized) in NK cells from controls and patients with COVID-19. Wilcoxon rank-sum test with Bonferroni-adjusted $P$ value indicated on top. $\mathbf{h}$ The selected functional alterations in DC cells in COVID-19 patients (Student's $t$ test; $* P<0.05$; $* * P<0.01$ ). i Circos plots of DC cells. The red bar on the outside indicates that the absolute value of $\log 2 \mathrm{FC}$ is greater than 0.5 . The blue bar suggests that the adjusted $P$ value is statistically significant $(<0.05)$. j Violin plots of $P K M$ expression (log-normalized) in DCs from controls and patients with COVID-19. Wilcoxon rank-sum test with Bonferroniadjusted $P$ value indicated on top. GST metabolism glycine, serine, threonine metabolism, $A A G$ metabolism alanine, aspartate and glutamate metabolism

$B$ cell activation and chemotaxis were upregulated in plasma cells in COVID-19 (Fig. 6d). Compared with T cells, plasma cells exhibited more significant metabolic alterations, as illustrated in the circos plot (Fig. 6e). Consistent with the increased metabolism score in glycolysis, OXPHOS, and fatty acid metabolism pathways in plasma cells (Fig. 2), most genes involved in these metabolic pathways were upregulated (Fig. 6f). Persistent neutralizing antibody production after viral infection is induced by long-lived plasma cells (LLPCs). Pyruvate-dependent respiration plays an important role in the function of LLPCs, and a deficiency in mitochondrial pyruvate import can cause a depletion of LLPCs, impairing vaccine-specific antibody production [58]. We found enhanced GPI, ENOI, and PKM expression in patients with COVID-19 (Fig. 6f), implying enhanced glucose catabolism that is likely followed by increased pyruvate generation. While there was no change in $M P C 2$ expression, a critical portion of the mitochondrial pyruvate carrier. In COVID-19 patients, plasma cells displayed increased OXPHOS (Fig. 6f), which was favorable for maximal antibody secretion [59]. The influence of other metabolic pathways on antibody production from plasma cells remains to be determined.
Metabolic alterations in fibroblasts and vascular smooth muscle cells

Studies have reported marked fibrotic changes in lung tissues from deceased COVID-19 patients [4, 5]. Here, we found that aging, proliferation, and migration were upregulated in alveolar fibroblasts, while apoptosis was decreased in COVID-19 patients (Fig. 7a). Consistent with metabolism scoring analysis (Fig. 2), the expression levels of most genes involved in glycolysis, pyruvate metabolism, OXPHOS, and inositol phosphate metabolism were increased in alveolar fibroblasts of COVID-19 patients as compared to healthy controls (Fig. 7b, c). The fibrotic response was associated with activation of the PLC/IP3/Ca2 ${ }^{+}$signaling pathway, which upregulates the expression levels of several matrix proteins, such as collagen A1 and fibronectin-1 [60]. The alveolar fibroblasts of patients with COVID-19 showed enhanced expression of PLCG2 compared to the control group (Fig. 7c). Unlike PLCG2, the expression level of phospholipase C epsilon 1 (PLCE1) was downregulated (Fig. 7c). Thus, in COVID-19, the precise association between PLC and fibroblast migration or fibrotic progression remains to be elucidated. Both aerobic glycolysis and pyruvate metabolism play important roles in fibroblast proliferation, myofibroblast differentiation, and fibrotic tissue alterations [61, 62]. $P K M$, $P G K 1$, and $E N O 1$ expression were found to be elevated in the alveolar fibroblasts of patients with COVID-19 (Fig. 7c), likely contributing to the aggravated fibrosis changes associated with this disease.

Pulmonary arterial hypertension (PAH) is positively correlated with the severity of COVID-19 [2]. The proliferation and migration of vascular smooth muscle cells (VSMCs) play a vital role in pulmonary vascular remodeling and PAH. Here, we found that VSMC aging, apoptosis, and proliferation were all upregulated in COVID-19 patients (Fig. 7d). Notably, correlation analysis illustrated that multiple metabolic pathways and metabolism-related signaling pathways were positively associated with VSMC aging, VSMC apoptosis, and VSMC proliferation (Fig. 7e). According to the DEGs exhibited in the circos plot, glycolysis and oxidative phosphorylation were the two major metabolic pathways altered in VSMCs in COVID-19 (Fig. 7f). A deficiency of $L D H A$ in vascular smooth muscle cells prevents VSMC proliferation and migration [63]. In hypoxiainduced PAH, the levels of lactate and hexokinase II were all increased. The inhibition of glycolysis could suppress VSMCs proliferation and migration, thus reversing vascular remodeling and relieving PAH [64]. Notably, several glycolysis-related enzymes showed remarkably increased expression in the VSMCs of patients with COVID-19, including $P K M, G A P D H, L D H A$, triosephosphate isomerase 1 (TPII), ENOI, PGKI (Fig. 7g). These alterations partially dictated 


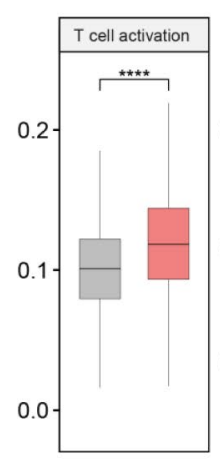

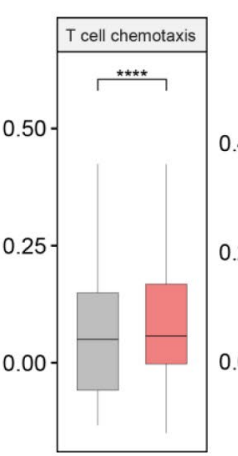

Control

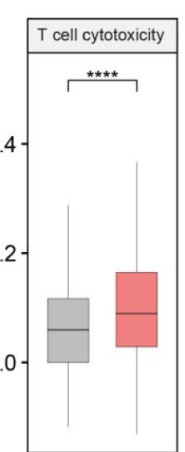

CoviD-19 b

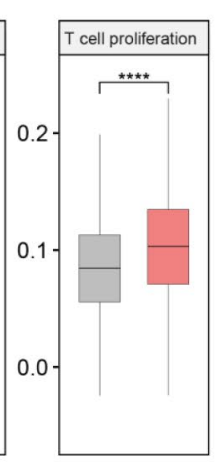

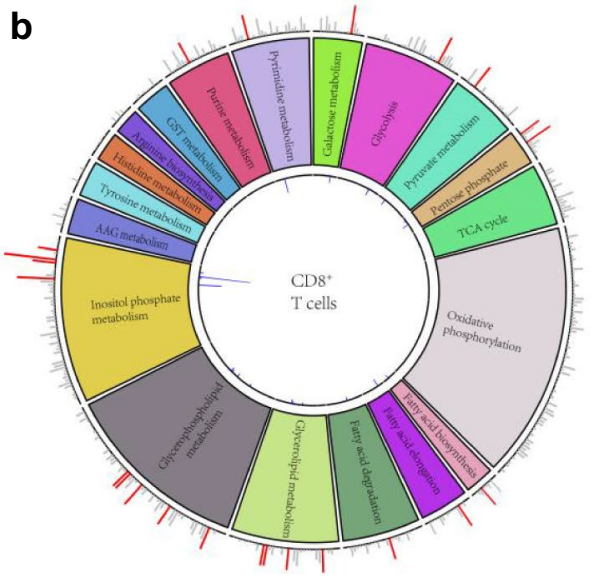

C

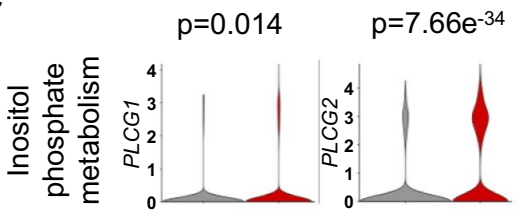

d
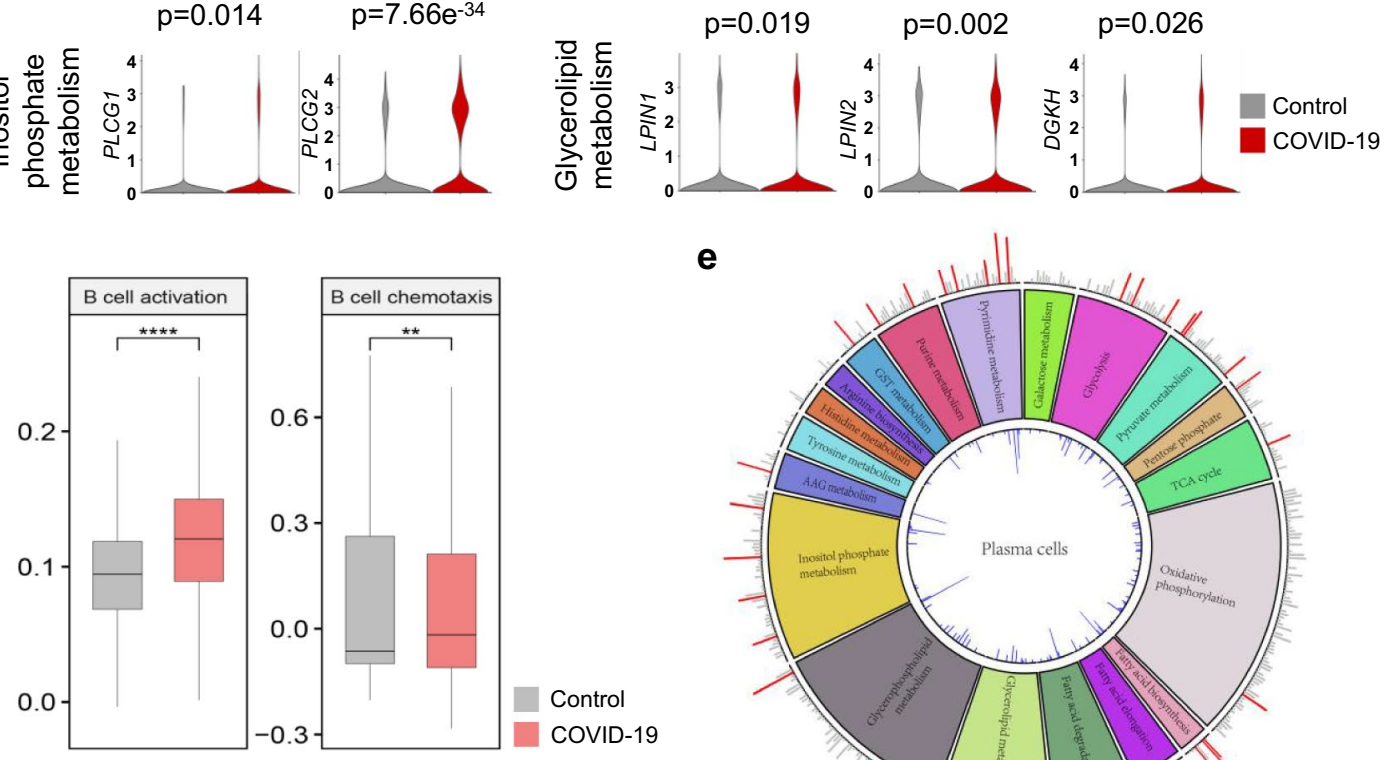

COVID-19

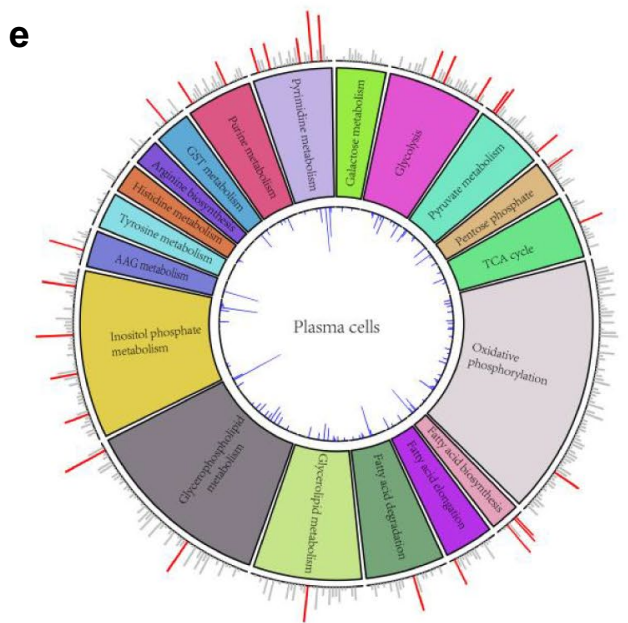

f

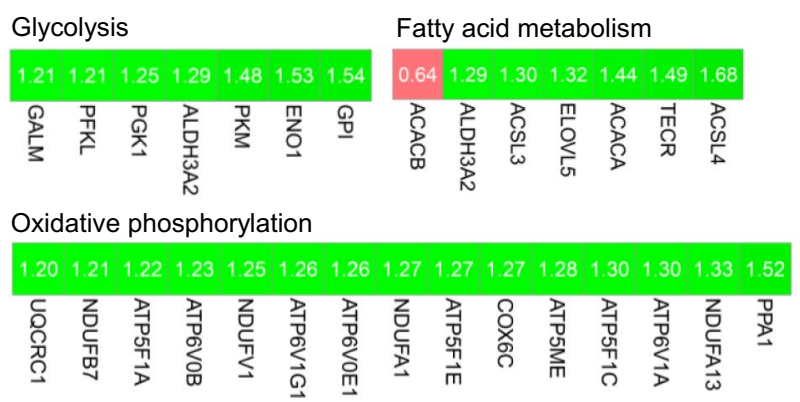

Fig. 6 The metabolic pathways that are altered in $\mathrm{CD}^{+} \mathrm{T}$ cells and plasma cells. a The selected functional alterations in $\mathrm{CD}^{+} \mathrm{T}$ cells in COVID-19 patients (Student's $t$ test; **** $P<0.0001$ ). b Circos plots of $\mathrm{CD}^{+} \mathrm{T}$ cells. The red bar on the outside indicates that the absolute value of $\log 2 \mathrm{FC}$ is greater than 0.5 . The blue bar suggests that the adjusted $P$ value is statistically significant $(<0.05)$. c Violin plots of PLCG1, PLCG2, LPIN1, LPIN2 and DGKH expression (log-normalized) in $\mathrm{CD}^{+} \mathrm{T}$ cells from controls and patients with COVID19. Wilcoxon rank-sum test with Bonferroni-adjusted $P$ value indicated on top. $\mathbf{d}$ The selected functional alterations in plasma cells in COVID-19 patients (Student's $t$ test; $* * P<0.01$; $* * * * P<0.0001$ ). e
Circos plots of plasma cells. The red bar on the outside indicates that the absolute value of $\log 2 \mathrm{FC}$ is greater than 0.5 . The blue bar suggests that the adjusted $P$ value is statistically significant $(<0.05)$. $\mathbf{f}$ Heat map demonstrating the fold changes of DEGs involved in a certain metabolic pathway in plasma cells. Green indicates the upregulated genes and red indicates the downregulated genes. The numbers in the squares represent the fold change of a certain gene, and darker colors indicate a larger fold change. GST metabolism glycine, serine, threonine metabolism, AAG metabolism alanine, aspartate and glutamate metabolism 
the metabolic basis of the development of PAH in COVID19. Several genes associated with OXPHOS, including ATP synthase F1 subunit epsilon (ATP5F1E), cytochrome c oxidase subunit $7 \mathrm{C}(C O X 7 C)$, cytochrome $\mathrm{c}$ oxidase subunit $6 \mathrm{C}$ (COX6C), and cytochrome c oxidase subunit 4I1 (COX4I1), were also upregulated in the COVID-19 group (Fig. 7g). Genes in glutathione metabolism and sphingolipid metabolism were significantly upregulated.

Lethal pneumonia pathogenesis is usually associated with respiratory failure, resulting from cytokine storm-induced airway obstruction or alveolar epithelial damage [65-69]. We found that upregulated glycolysis, OXPHOS, glutathione metabolism, and sphingolipid metabolism may promote AT1 cell aging. Enhanced glycolysis in AT2 cells may impair the differentiation of AT2 cells into AT1 cells. Glutathione metabolism connects with AT2 aging. Once alterations in these metabolism processes occur in AT1 and AT2 cells after SARS-CoV-2 infection, alveolar epithelial damage may develop leading to lethal outcomes. Second, upregulated glycolysis and inositol phosphate metabolism in lung fibroblast cells contribute to lung fibrotic progression by promoting fibroblast cell activation and differentiation. Third, upregulated OXPHOS, inositol phosphate metabolism and glycolysis contribute to activation and pro-inflammatory phenotype of macrophages, respectively. Upregulation of oxidative phosphorylation, glutathione metabolism and sphingolipid metabolism are associated with vascular smooth muscle cell aging or apoptosis that may increase risk of pulmonary arterial hypertension of COVID-19 patients. Altogether, multiple altered metabolic pathways in various cell types relate to over-activated immune responses and lung tissue damage in critical COVID-19 patients (Fig. 8).

\section{Discussion}

Patients with COVID-19 display multiple tissue pathologies that are usually associated with alterations in cellular function. A potent immune defense against SARS-CoV-2 infection demands both the innate (physical epithelial barrier and innate immune cells, molecules) and adaptive immune responses. Immune dysregulation has been reported during COVID-19, including the presence of defective dendritic cell and $\mathrm{T}$ cell responses, impaired IFN response, lymphopenia, and cytokine storm [52, 70, 71]. However, the potential cellular metabolism-related mechanisms of immune dysregulation in COVID-19 are not yet fully understood. By analyzing the snRNA-seq data in the lungs obtained from deceased COVID-19 patients, we comprehensively revealed the transcriptional changes of genes involved in 35 metabolic pathways and 12 metabolism-related signaling pathways across various cell types, presenting evidence of cellular metabolic changes after SARS-CoV-2 infection (Fig. 8).
As a vital component of the innate immune system, the physical epithelial barrier exerts a critical host defense against invasion by environmental pathogens. The epithelial barrier function is damaged in COVID-19 [72]. Here, we report the metabolic basis of epithelial cell aging, defective airway mucociliary clearance, impaired alveolar repair, and damaged surfactant hemostasis after SARSCoV-2 infection. The metabolic pathways involved in these processes include glycolysis, fatty acid metabolism, glutathione metabolism, and inositol phosphate metabolism, and several signaling pathways, including cAMP, cGMPPKG, and MAPK signaling pathways. However, only a few metabolism-related genes changed in airway basal cells (See Figure S1 in the Supplementary Material, Fig. 2), consistent with the fact that basal cells are not affected by SARS-CoV-2 [73]. Enhanced inositol phosphate metabolism was observed in club cells, accompanied by increased expression of PLCG2 and PIK3CA. Both PLC and PI3K are associated with interleukin-8 (IL-8) expression in airway epithelial cells, and IL-8 is a potent chemokine for neutrophils [74-76]. Neutrophil-IL-8-axis is associated with the immuno-thrombotic state observed in severe COVID-19 patients [77]. Neutrophils drive necro-inflammation and are positively related to mortality in COVID19 patients with comorbidities, such as cardiovascular disease and diabetes [78]. The decreased expression of multiple airway mucins indicated impaired mucociliary clearance in patients with COVID-19, which could cause bacterial traps and infections in the airways, promoting disease severity and even death. This finding contradicts other reports that showed pathological mucus hypersecretion in patients with COVID-19, resulting in airway obstruction or respiratory distress [79]. Altogether, these data suggest that both a deficiency and overproduction of mucins contribute to COVID-19 progression. Recent research demonstrates that senescence is a pathological characteristic of the lungs after SARS-CoV-2 infection, which in turn contributes to lung aging and related disorders [80]. Concordantly, we observed significant upregulated aging in epithelial cells, including club cells, goblet cells, AT1 cells and AT2 cells. Metabolic processes, such as OXPHOS, sphingolipid metabolism, and glutathione metabolism, may be the metabolic basis of aging in these cells.

Other innate immune cells, including macrophages, NK cells, and DCs, also present several metabolism-related alterations, impairing effective virus elimination. Alveolar macrophages from patients with COVID-19 may present with a more active state [27]. Metabolic changes in alveolar macrophages, including glycolysis, OXPHOS, inositol phosphate metabolism, and glycerolipid metabolism, may affect their functions, such as migration, phagocytosis, and inflammatory phenotype. These alterations 
a

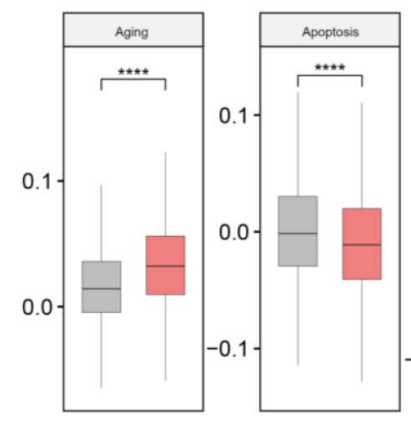

C

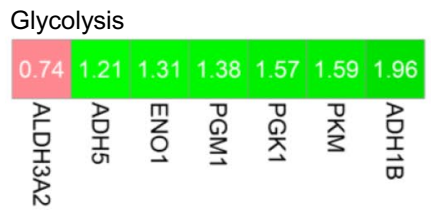

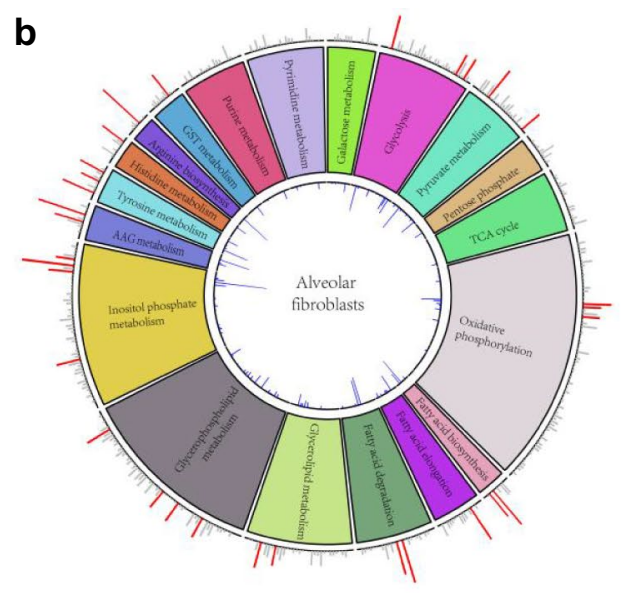

Pyruvate metabolism

$$
\begin{aligned}
& \begin{array}{llllllll}
0.74 & 1.21 & 1.24 & 1.30 & 1.59 & 1.96
\end{array}
\end{aligned}
$$

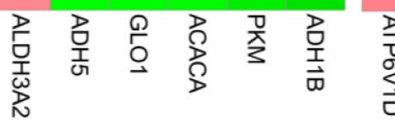

e

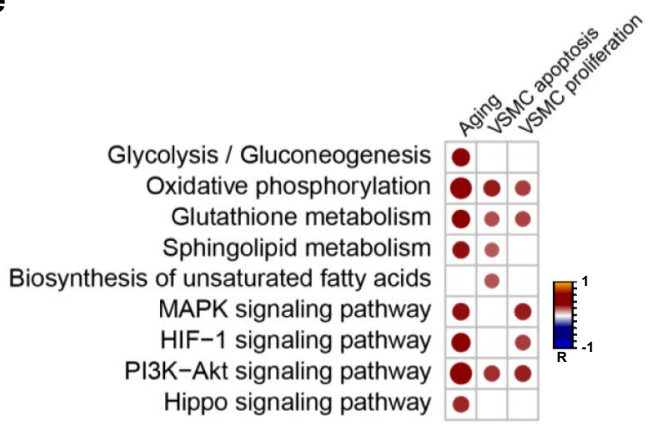

d

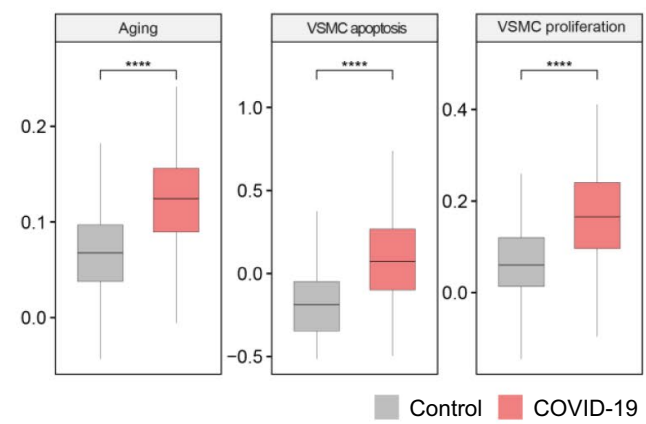

Control $\square$ COVID-19
Oxidative phosphorylation

Inositol phosphate metabolism

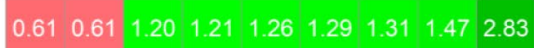

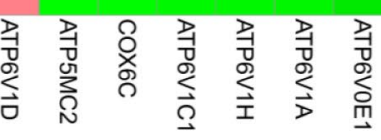

f

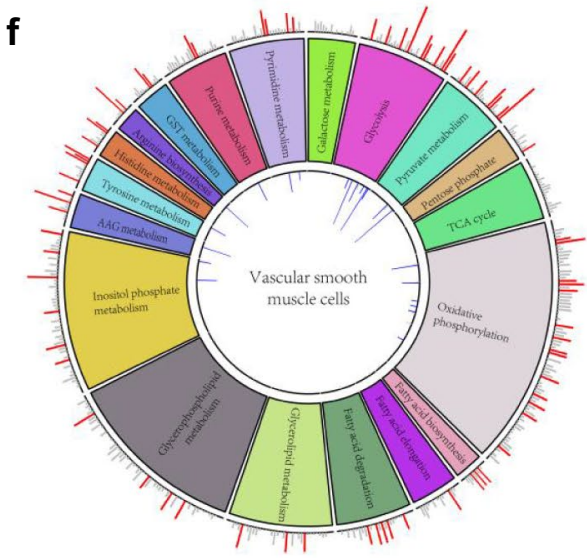

g

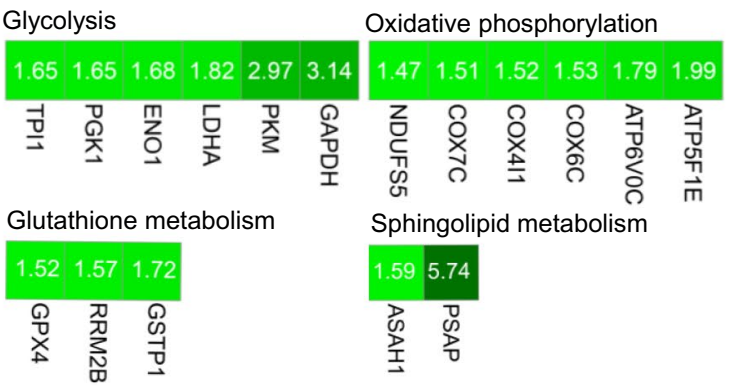


4Fig. 7 The metabolic pathways that are altered in alveolar fibroblasts and vascular smooth muscle cells (VSMCs). a The selected functional alterations in alveolar fibroblasts in COVID-19 patients (Student's $t$-test; $* * * * P<0.0001)$. b Circos plots of alveolar fibroblasts. The red bar on the outside indicates that the absolute value of $\log 2 \mathrm{FC}$ is greater than 0.5 . The blue bar suggests that the adjusted $P$ value is statistically significant $(<0.05)$. c Heat map demonstrating the fold changes of DEGs involved in a certain metabolic pathway in alveolar fibroblasts. Green indicates the upregulated genes and red indicates the downregulated genes. The numbers in the squares represent the fold change of a certain gene, and darker colors indicate a larger fold change. $\mathbf{d}$ The selected functional alterations in VSMCs in COVID19 patients (Student's $t$-test; $* * * * P<0.0001$ ). e The Pearson correlations between the scores of VSMCs function and the metabolic pathways. " $R$ " indicates the Pearson correlation coefficient; only dots representing correlations with $|R|>0.2$ and $P$ value $<0.05$ are displayed. f Circos plots of VSMCs. The red bar on the outside indicates that the absolute value of $\log 2 \mathrm{FC}$ is greater than 0.5 . The blue bar suggests that the adjusted $P$ value is statistically significant $(<0.05)$. g Heat map demonstrating the fold changes of DEGs involved in a certain metabolic pathway in alveolar fibroblasts and VSMCs, respectively. Green indicates the upregulated genes and red indicates the downregulated genes. The numbers in the squares represent the fold change of a certain gene, and darker colors indicate a larger fold change. GST metabolism glycine, serine, threonine metabolism, $A A G$ metabolism alanine, aspartate and glutamate metabolism

likely contribute to virus clearance, but over-activated macrophages may also simultaneously aggravate inflammatory responses and tissue injury. Research has reported the impaired cytotoxicity of NK cells in COVID-19 [48]. Increased glycolysis, glycerolipid metabolism, and inositol phosphate metabolism may contribute to enhanced NK cell functions, including degranulation and cytotoxicity, as well as aggravated inflammatory damage. DCs are important antigen-presenting and type 1 IFN-generating cells. Virus infection and replication can put DCs under the stress of extra energy demand [18]. Except for $P K M$, ATP6VOA1, ATP5F $1 C$, and ALDH2, most genes involved in glycolysis, oxidative phosphorylation, and fatty acid metabolism remained unchanged in terms of expression after SARS-CoV-2 infection. The contradiction between increased energy demand and the unavailability of sufficient energy supply during infection may finally lead to $\mathrm{DC}$ apoptosis and impaired antigen presentation and $\mathrm{T}$ cell function [18].

Elderly COVID- 19 patients presented damaged CD $8^{+} \mathrm{T}$ cell cytotoxic responses compared with younger patients, and this may partially explain the more serious clinical manifestations and higher mortality of elderly patients [81]. The enhanced cytotoxicity of $\mathrm{CD} 8^{+} \mathrm{T}$ cells may be partially attributed to DAG signaling mediated by metabolic changes in the inositol phosphate and glycerolipid metabolism pathways. The enhanced glucose catabolism and OXPHOS in COVID-19-induced plasma cells likely contributed to maximal antibody production. Titers of neutralizing antibodies were positively correlated with COVID-19 disease severity. Patients in the intensive care unit exhibited higher antibody titers, but asymptomatic or individuals managed exclusively in outpatient care showed no or lower antibody titers [82]. Antibody-mediated immune function may induce both protective and pathological outcomes. The robust humoral response can be a characteristic of excessive immune activation and correlates with the levels of inflammatory cytokines and chemokines, as observed in patients with severe COVID-19 [83]. We observed that the glycolysis and OXPHOS pathways favoring neutralizing antibody production are upregulated in deceased COVID19 patients. However, if patients failed to form long-lasting plasma cells, SARS-CoV-2 infection-induced damage would not be stopped [18].

Extensive pulmonary fibrosis has been identified via histological and radiological findings in the lung tissue of deceased COVID-19 patients [84-88]. Enhanced glycolysis and inositol phosphate metabolism in fibroblasts may promote fibrogenesis in severe COVID-19. PLC-mediated inositol phosphate metabolism was associated with matrix protein secretion and fibroblast chemotaxis $[60,89]$. Furthermore, the inhibition of aerobic glycolysis was shown to attenuate renal fibrosis and myofibroblast activation through the dephosphorylation of pyruvate kinase M2 (PKM2) [62]. A deficiency of PKM2 in fibroblasts inhibits fibroblast proliferation [61]. These data support the concept that targeting cellular metabolism may be valid for addressing the fibrosis observed in COVID-19 and other respiratory diseases.

We previously observed that phosphatidylinositol and lysophosphatidylinositol levels are increased in serum of recovered severe acute respiratory syndrome-coronavirus patients 12 years after infection [90]. In influenza A virusinfected C57BL/6 mice, lung epithelial cell metabolism shifted to glycolysis and glycolysis inhibitor, 2-deoxy-Dglucose, exacerbated lung dysfunction [91]. The upregulation of the pentose phosphate pathway was proposed to produce nucleotides required for influenza viral replication [92]. Quantitative proteomics analysis of primary human bronchial epithelial cells showed upregulated glycolysis and glutaminolysis after infection with human H1N1 influenza virus [93]. Human respiratory syncytial virus (HRSV) ensured the supply of energy and structural components for virus replication by upregulation of glycolysis and citric acid cycle components, nucleotides, amino acids, and nucleotide sugars [94]. Glucose metabolism, lipid metabolism and inositol phosphate metabolism represent the most significant changed metabolic pathways in many respiratory virus infections. However, there are few studies to reveal metabolic alterations in response to respiratory viruses at cellular basis. Only by analyzing the metabolic changes in various cells in respiratory virus infections can we deeply understand the effectiveness and end of host immunity. In this study, amino acid metabolism was found altered in many cell types in the 


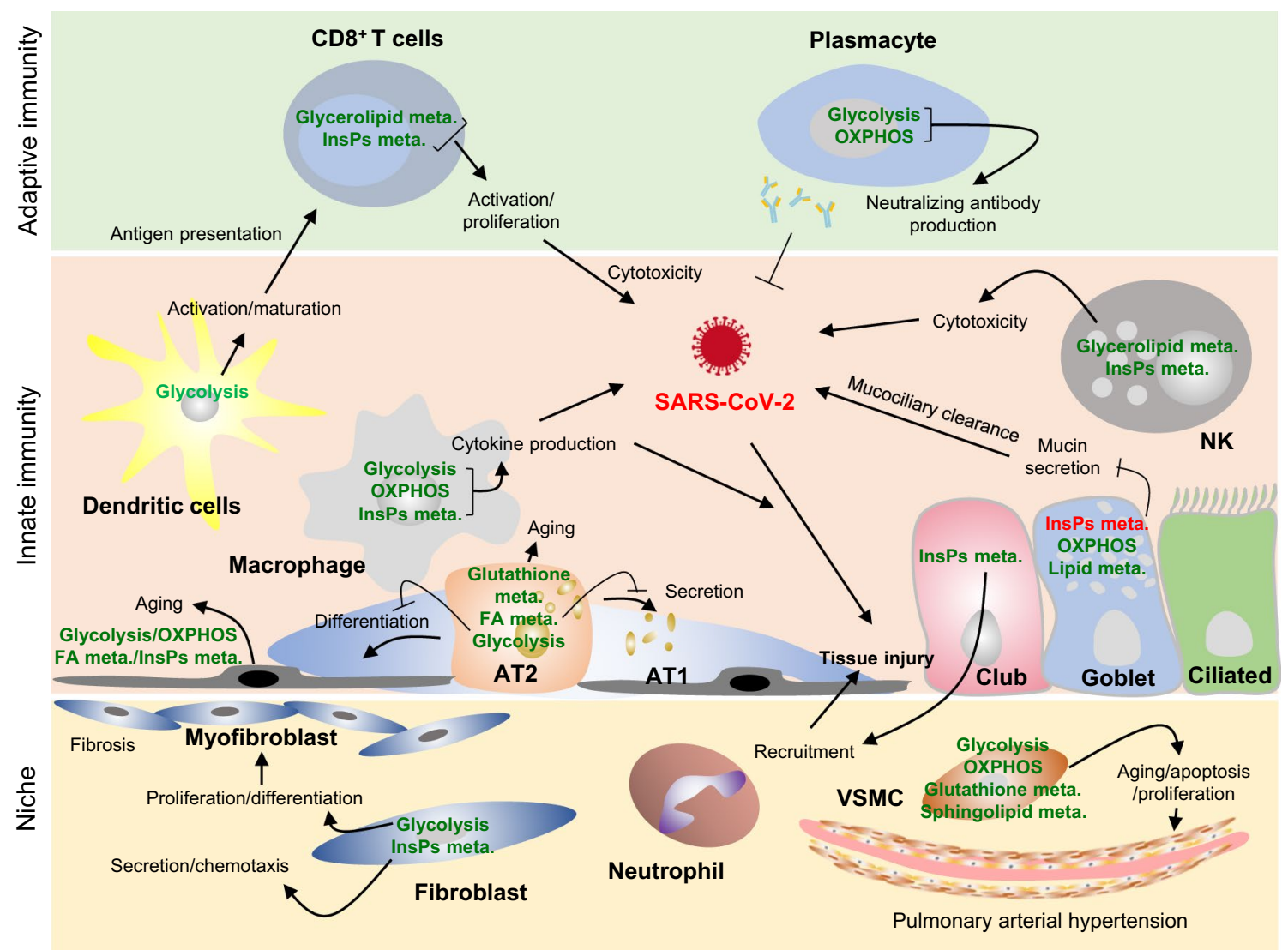

Fig. 8 Schematic illustration of cellular metabolism during SARSCoV-2 infection. After SARS-CoV-2 infection, lung cells exhibited remarkable metabolic changes, including that in glycolysis, oxidative phosphorylation, lipid metabolism, and inositol phosphate metabolism pathways. These metabolic pathways influence the host defense against SARS-CoV-2 infection, including mucociliary clearance of airway epithelial cells, surfactant hemostasis of alveolar epithelial cells, cytotoxicity of $\mathrm{CD} 8^{+} \mathrm{T}$ cells and NK cells, neutralizing antibody production by plasma cells, and the phagocytosis of macrophages. Metabolic changes in fibroblasts are related to cellular proliferation, chemotaxis, matrix secretion, and differentiation into myofibroblasts, leading to fibrogenesis. Glycolysis, OXPHOS, glutathione metabolism may boost the proliferation and migration capability of vascular smooth muscle cells, resulting in pulmonary arterial hypertension. InsPs meta. Inositol phosphate metabolism, FA meta. Fatty acid metabolism, OXPHOS oxidative phosphorylation, VSMC vascular smooth muscle cell, $N K$ natural killer cell lung after SARS-CoV-2 infection. These metabolic changes have not been reported in other respiratory virus infections and are worthy of further study.

This study had several limitations. First, some genes associated with cellular metabolism were missed in the snRNAseq data because of the sequencing technology used. Second, snRNA-seq analysis in this study is only suggestive because enzyme activity is regulated not only by transcription levels but also by covalent modification, such as phosphorylation and acetylation. Thus, a multi-omics analysis, including transcriptomics, proteomics, and metabolomics, could be performed to examine the metabolic changes in lung immunity after SARS-CoV-2 infection. We have provided several hints about host cell metabolic alterations in COVID-19, and their subsequent biological effects will be verified in future studies.
Supplementary Information The online version contains supplementary material available at https://doi.org/10.1007/s00430-021-00727-0.

Funding This study was supported by the National Natural Science Foundation of China (81773394, 82070001, 81970001), Natural Science Foundation of Tianjin (18ZXDBSY00150). The funders had no role in the study design, data collection and analysis, decision to publish, or preparation of the manuscript.

Data availability The single-nucleus RNA-sequencing (snRNA-seq) data of lung tissues from 19 deceased COVID-19 patients (12 men and 7 women) and 7 controls ( 4 men and 3 women) were obtained from the GEO database (https://www.ncbi.nlm.nih.gov/geo/), accession number: GSE171524.

\section{Declarations}

Conflict of interest The authors declare no conflict of interest. 


\section{References}

1. Pelaia C, Calabrese C, Garofalo E et al (2021) Therapeutic role of tocilizumab in SARS-CoV-2-induced cytokine storm: rationale and current evidence. Int J Mol Sci 22(6):3059

2. Pagnesi M, Baldetti L, Beneduce A et al (2020) Pulmonary hypertension and right ventricular involvement in hospitalised patients with COVID-19. Heart 106(17):1324-1331

3. Bradley BT, Maioli H, Johnston R et al (2020) Histopathology and ultrastructural findings of fatal COVID-19 infections in Washington State: a case series. Lancet 396(10247):320-332

4. Grillo F, Barisione E, Ball L et al (2021) Lung fibrosis: an undervalued finding in COVID-19 pathological series. Lancet Infect Dis 21(4):e72

5. Carsana L, Sonzogni A, Nasr A et al (2020) Pulmonary postmortem findings in a series of COVID-19 cases from northern Italy: a two-centre descriptive study. Lancet Infect Dis 20(10): $1135-1140$

6. Wang Y, Tang N (2021) The diversity of adult lung epithelial stem cells and their niche in homeostasis and regeneration. Sci China Life Sci 64:2045

7. Li X, Zhao F, Wang A et al (2021) Role and mechanisms of autophagy in lung metabolism and repair. Cell Mol Life Sci 78(12):5051-5068

8. Hou YJ, Okuda K, Edwards CE et al (2020) SARS-CoV-2 reverse genetics reveals a variable infection gradient in the respiratory tract. Cell 182(2):429-446

9. Zhang H, Rostami MR, Leopold PL et al (2020) Expression of the SARS-CoV-2 ACE2 receptor in the human airway epithelium. Am J Respir Crit Care Med 202(2):219-229

10. Speranza E, Williamson BN, Feldmann F et al (2021) Singlecell RNA sequencing reveals SARS-CoV-2 infection dynamics in lungs of African green monkeys. Sci Transl Med 13(578)

11. Ricci D, Etna MP, Rizzo F et al (2021) Innate immune response to SARS-CoV-2 infection: from cells to soluble mediators. Int J Mol Sci 22(13):7017

12. Parackova Z, Zentsova I, Bloomfield M et al (2020) Disharmonic inflammatory signatures in COVID-19: augmented neutrophils' but impaired monocytes' and dendritic cells' responsiveness. Cells 9(10):2206

13. Schultze JL, Aschenbrenner AC (2021) COVID-19 and the human innate immune system. Cell 184(7):1671-1692

14. Rydyznski Moderbacher C, Ramirez SI, Dan JM et al (2020) Antigen-specific adaptive immunity to SARS-CoV-2 in acute COVID-19 and associations with age and disease severity. Cell 183(4):996-1012

15. DiPiazza AT, Graham BS, Ruckwardt TJ (2021) T cell immunity to SARS-CoV-2 following natural infection and vaccination. Biochem Biophys Res Commun 538:211-217

16. Li X, Wu J, Sun X et al (2020) Autophagy reprograms alveolar progenitor cell metabolism in response to lung injury. Stem Cell Reports 14(3):420-432

17. Li K, Li M, Li W et al (2019) Airway epithelial regeneration requires autophagy and glucose metabolism. Cell Death Dis 10(12):875

18. Kumar V (2021) How could we forget immunometabolism in SARS-CoV2 infection or COVID-19? Int Rev Immunol 40(1-2):72-107

19. Codo AC, Davanzo GG, Monteiro LB et al (2020) Elevated glucose levels favor SARS-CoV-2 infection and monocyte response through a HIF-1alpha/glycolysis-dependent axis. Cell Metab 32(3):437-446

20. Loftus RM, Assmann N, Kedia-Mehta N et al (2018) Amino acid-dependent cMyc expression is essential for NK cell metabolic and functional responses in mice. Nat Commun 9(1):2341
21. Widjaja CE, Olvera JG, Metz PJ et al (2017) Proteasome activity regulates $\mathrm{CD} 8+\mathrm{T}$ lymphocyte metabolism and fate specification. J Clin Invest 127(10):3609-3623

22. Mahrooz A, Muscogiuri G, Buzzetti R et al (2021) The complex combination of COVID-19 and diabetes: pleiotropic changes in glucose metabolism. Endocrine 72(2):317-325

23. McElvaney OJ, McEvoy NL, McElvaney OF et al (2020) Characterization of the inflammatory response to severe COVID-19 illness. Am J Respir Crit Care Med 202(6):812-821

24. Hu X, Chen D, Wu L et al (2020) Declined serum high density lipoprotein cholesterol is associated with the severity of COVID-19 infection. Clin Chim Acta 510:105-110

25. Morawietz H, Julius U, Bornstein SR (2020) Cardiovascular diseases, lipid-lowering therapies and European registries in the COVID-19 pandemic. Cardiovasc Res 116(10):e122-e125

26. Paez-Franco JC, Torres-Ruiz J, Sosa-Hernandez VA et al (2021) Metabolomics analysis reveals a modified amino acid metabolism that correlates with altered oxygen homeostasis in COVID19 patients. Sci Rep 11(1):6350

27. Melms JC, Biermann J, Huang $\mathrm{H}$ et al (2021) A molecular single-cell lung atlas of lethal COVID-19. Nature 595(7865):114-119

28. Rostami MR, LeBlanc MG, Strulovici-Barel Y et al (2021) Smoking shifts human small airway epithelium club cells toward a lesser differentiated population. NPJ Genom Med $6(1): 73$

29. Hiemstra PS, Bourdin A (2014) Club cells, CC10 and self-control at the epithelial surface. Eur Respir J 44(4):831-832

30. Santos AF, Povoa P, Paixao P et al (2021) Changes in glycolytic pathway in SARS-COV 2 infection and their importance in understanding the severity of COVID-19. Front Chem 9:685196

31. Irvine RF, Schell MJ (2001) Back in the water: the return of the inositol phosphates. Nat Rev Mol Cell Biol 2(5):327-338

32. Sunderhauf A, Hicken M, Schlichting H et al (2021) Loss of mucosal $\mathrm{p} 32 / \mathrm{gC} 1 \mathrm{qR} / \mathrm{HABP} 1$ triggers energy deficiency and impairs goblet cell differentiation in ulcerative colitis. Cell Mol Gastroenterol Hepatol 12(1):229-250

33. Kim KC, McCracken K, Lee BC et al (1997) Airway goblet cell mucin: its structure and regulation of secretion. Eur Respir $\mathbf{J}$ 10(11):2644-2649

34. Chatterjee M, van Putten JPM, Strijbis K (2020) Defensive properties of mucin glycoproteins during respiratory infections-relevance for SARS-CoV-2. mBio 11(6)

35. Wang S, Yao X, Ma S et al (2021) A single-cell transcriptomic landscape of the lungs of patients with COVID-19. Nat Cell Biol 23(12):1314-1328

36. Choi J, Park JE, Tsagkogeorga G et al (2020) Inflammatory signals induce AT2 cell-derived damage-associated transient progenitors that mediate alveolar regeneration. Cell Stem Cell 27(3):366-382

37. Whitsett JA, Wert SE, Weaver TE (2015) Diseases of pulmonary surfactant homeostasis. Annu Rev Pathol 10:371-393

38. McCormack FX, Whitsett JA (2002) The pulmonary collectins, SP-A and SP-D, orchestrate innate immunity in the lung. J Clin Invest 109(6):707-712

39. Peca D, Cutrera R, Masotti A et al (2015) ABCA3, a key player in neonatal respiratory transition and genetic disorders of the surfactant system. Biochem Soc Trans 43(5):913-919

40. Wang C, Xie J, Zhao L et al (2020) Alveolar macrophage dysfunction and cytokine storm in the pathogenesis of two severe COVID-19 patients. EBioMedicine 57:102833

41. Kissing S, Saftig P, Haas A (2018) Vacuolar ATPase in phago(lyso)some biology. Int J Med Microbiol 308(1):58-67

42. Perez MC, Bodine PV, Leiva MC et al (2002) Signal transduction pathways involved in macrophage migration induced by peritoneal fluid chemotactic factors in stages I and II endometriosis. Fertil Steril 77(6):1261-1268 
43. Shen B, Yi X, Sun Y et al (2020) Proteomic and metabolomic characterization of COVID-19 patient sera. Cell 182(1):59-72

44. Trent CM, Yu S, Hu Y et al (2014) Lipoprotein lipase activity is required for cardiac lipid droplet production. J Lipid Res 55(4):645-658

45. Lee CH, Kang K, Mehl IR et al (2006) Peroxisome proliferatoractivated receptor delta promotes very low-density lipoproteinderived fatty acid catabolism in the macrophage. Proc Natl Acad Sci U S A 103(7):2434-2439

46. Barish GD, Atkins AR, Downes M et al (2008) PPARdelta regulates multiple proinflammatory pathways to suppress atherosclerosis. Proc Natl Acad Sci U S A 105(11):4271-4276

47. Zhang Y, Yuan D, Li Y et al (2021) Paraquat promotes acute lung injury in rats by regulating alveolar macrophage polarization through glycolysis. Ecotoxicol Environ Saf 223:112571

48. van Eeden C, Khan L, Osman MS et al (2020) Natural killer cell dysfunction and its role in COVID-19. Int J Mol Sci 21(17):6351

49. Sheppard S, Santosa EK, Lau CM et al (2021) Lactate dehydrogenase A-dependent aerobic glycolysis promotes natural killer cell anti-viral and anti-tumor function. Cell Rep 35(9):109210

50. Caraux A, Kim N, Bell SE et al (2006) Phospholipase C-gamma2 is essential for NK cell cytotoxicity and innate immunity to malignant and virally infected cells. Blood 107(3):994-1002

51. Naik SH, Sathe P, Park HY et al (2007) Development of plasmacytoid and conventional dendritic cell subtypes from single precursor cells derived in vitro and in vivo. Nat Immunol 8(11):1217-1226

52. Zhou R, To KK, Wong YC et al (2020) Acute SARS-CoV-2 infection impairs dendritic cell and $\mathrm{T}$ cell responses. Immunity 53(4):864-877

53. Jin X, Zhang W, Wang Y et al (2020) Pyruvate kinase M2 promotes the activation of dendritic cells by enhancing IL-12p35 expression. Cell Rep 31(8):107690

54. Liu C, Zheng M, Wang T et al (2018) PKM2 is required to activate myeloid dendritic cells from patients with severe aplastic anemia. Oxid Med Cell Longev 2018:1364165

55. Zhang W, Trible RP, Zhu M et al (2000) Association of Grb2, Gads, and phospholipase C-gamma 1 with phosphorylated LAT tyrosine residues. Effect of LAT tyrosine mutations on $\mathrm{T}$ cell angigen receptor-mediated signaling. J Biol Chem 275(30):23355-23361

56. Yablonski D, Kuhne MR, Kadlecek T et al (1998) Uncoupling of nonreceptor tyrosine kinases from PLC-gamma1 in an SLP76-deficient T cell. Science 281(5375):413-416

57. Shin J, O'Brien TF, Grayson JM et al (2012) Differential regulation of primary and memory CD8 T cell immune responses by diacylglycerol kinases. J Immunol 188(5):2111-2117

58. Lam WY, Becker AM, Kennerly KM et al (2016) Mitochondrial pyruvate import promotes long-term survival of antibody-secreting plasma cells. Immunity 45(1):60-73

59. Price MJ, Patterson DG, Scharer CD et al (2018) Progressive upregulation of oxidative metabolism facilitates plasmablast differentiation to a T-independent antigen. Cell Rep 23(11):3152-3159

60. Mukherjee S, Duan F, Kolb MR et al (2013) Platelet derived growth factor-evoked $\mathrm{Ca} 2+$ wave and matrix gene expression through phospholipase $\mathrm{C}$ in human pulmonary fibroblast. Int $\mathrm{J}$ Biochem Cell Biol 45(7):1516-1524

61. Ye Y, Xu L, Ding H et al (2021) Pyruvate kinase M2 mediates fibroblast proliferation to promote tubular epithelial cell survival in acute kidney injury. FASEB J 35(7):e21706

62. Ding H, Jiang L, Xu J et al (2017) Inhibiting aerobic glycolysis suppresses renal interstitial fibroblast activation and renal fibrosis. Am J Physiol Renal Physiol 313(3):F561-F575
63. Kim JH, Bae KH, Byun JK et al (2017) Lactate dehydrogenase-A is indispensable for vascular smooth muscle cell proliferation and migration. Biochem Biophys Res Commun 492(1):41-47

64. Chen F, Wang H, Lai J et al (2018) 3-Bromopyruvate reverses hypoxia-induced pulmonary arterial hypertension through inhibiting glycolysis: in vitro and in vivo studies. Int J Cardiol 266:236-241

65. Gupta SK, Srivastava M, Minocha R et al (2021) Alveolar regeneration in COVID-19 patients: a network perspective. Int J Mol Sci 22(20): 11279

66. Sadegh Beigee F, Pourabdollah Toutkaboni M, Khalili N et al (2020) Diffuse alveolar damage and thrombotic microangiopathy are the main histopathological findings in lung tissue biopsy samples of COVID-19 patients. Pathol Res Pract 216(10):153228

67. Tian $\mathrm{W}$, Zhang $\mathrm{N}$, Jin R et al (2020) Immune suppression in the early stage of COVID-19 disease. Nat Commun 11(1):5859

68. Blanco-Melo D, Nilsson-Payant BE, Liu WC et al (2020) Imbalanced host response to SARS-CoV-2 drives development of COVID-19. Cell 181(5):1036-1045

69. Zhao Y, Sui L, Wu P et al (2021) A dual-role of SARS-CoV-2 nucleocapsid protein in regulating innate immune response. Signal Transduct Target Ther 6(1):331

70. Esmaeilzadeh A, Elahi R (2021) Immunobiology and immunotherapy of COVID-19: a clinically updated overview. J Cell Physiol 236(4):2519-2543

71. Files JK, Boppana S, Perez MD et al (2021) Sustained cellular immune dysregulation in individuals recovering from SARSCoV-2 infection. J Clin Invest 131(1)

72. Robinot R, Hubert M, de Melo GD et al (2021) SARS-CoV-2 infection induces the dedifferentiation of multiciliated cells and impairs mucociliary clearance. Nat Commun 12(1):4354

73. Bridges JP, Vladar EK, Huang H et al (2021) Respiratory epithelial cell responses to SARS-CoV-2 in COVID-19. Thorax

74. Yoon JH, Jeong SH, Hong JH (2015) The effect of therapeutic blockades of dust particles-induced $\mathrm{Ca}(2)(+)$ signaling and proinflammatory cytokine IL-8 in human bronchial epithelial cells. Mediators Inflamm 2015:843024

75. Newcomb DC, Sajjan U, Nanua S et al (2005) Phosphatidylinositol 3-kinase is required for rhinovirus-induced airway epithelial cell interleukin-8 expression. J Biol Chem 280(44):36952-36961

76. Bentley JK, Newcomb DC, Goldsmith AM et al (2007) Rhinovirus activates interleukin-8 expression via a Src/p110beta phosphatidylinositol 3-kinase/Akt pathway in human airway epithelial cells. J Virol 81(3):1186-1194

77. Kaiser R, Leunig A, Pekayvaz K et al (2021) Self-sustaining IL-8 loops drive a prothrombotic neutrophil phenotype in severe COVID-19. JCI Insight 6(18)

78. Tomar B, Anders HJ, Desai J et al (2020) Neutrophils and neutrophil extracellular traps drive necroinflammation in COVID-19. Cells 9(6): 1383

79. Yin W, Cao W, Zhou G et al (2021) Analysis of pathological changes in the epithelium in COVID-19 patient airways. ERJ Open Res 7(2):00690-02020

80. Wang S, Yao X, Ma S et al (2021) A single-cell transcriptomic landscape of the lungs of patients with COVID-19. Nat Cell Biol 23:1314

81. Westmeier J, Paniskaki K, Karakose Z et al (2020) Impaired cytotoxic CD8(+) T cell response in elderly COVID-19 patients. mBio 11(5)

82. Legros V, Denolly S, Vogrig M et al (2021) A longitudinal study of SARS-CoV-2-infected patients reveals a high correlation between neutralizing antibodies and COVID-19 severity. Cell Mol Immunol 18(2):318-327

83. Wang K, Long QX, Deng HJ et al (2021) Longitudinal dynamics of the neutralizing antibody response to severe acute respiratory 
syndrome coronavirus 2 (SARS-CoV-2) infection. Clin Infect Dis 73(3): e531-e539

84. Shi H, Han X, Jiang N et al (2020) Radiological findings from 81 patients with COVID-19 pneumonia in Wuhan, China: a descriptive study. Lancet Infect Dis 20(4):425-434

85. Schwensen HF, Borreschmidt LK, Storgaard M et al (2020) Fatal pulmonary fibrosis: a post-COVID-19 autopsy case. J Clin Pathol 74:400

86. Chen JY, Qiao K, Liu F et al (2020) Lung transplantation as therapeutic option in acute respiratory distress syndrome for coronavirus disease 2019-related pulmonary fibrosis. Chin Med J (Engl) 133(12):1390-1396

87. Xu Z, Shi L, Wang Y et al (2020) Pathological findings of COVID-19 associated with acute respiratory distress syndrome. Lancet Respir Med 8(4):420-422

88. Schaller T, Hirschbuhl K, Burkhardt K et al (2020) Postmortem examination of patients with COVID-19. JAMA 323(24):2518-2520

89. Martins M, Warren S, Kimberley C et al (2012) Activity of PLCepsilon contributes to chemotaxis of fibroblasts towards PDGF. J Cell Sci 125(Pt 23):5758-5769
90. Wu Q, Zhou L, Sun X et al (2017) Altered lipid metabolism in recovered SARS patients twelve years after infection. Sci Rep 7(1):9110

91. Nolan KE, Baer LA, Karekar P et al (2021) Metabolic shifts modulate lung injury caused by infection with H1N1 influenza A virus. Virology 559:111-119

92. Erlich JR, To EE, Liong S et al (2020) Targeting evolutionary conserved oxidative stress and immunometabolic pathways for the treatment of respiratory infectious diseases. Antioxid Redox Signal 32(13):993-1013

93. Smallwood HS, Duan S, Morfouace M et al (2017) Targeting metabolic reprogramming by influenza infection for therapeutic intervention. Cell Rep 19(8):1640-1653

94. Martin-Vicente M, Gonzalez-Riano C, Barbas C et al (2020) Metabolic changes during respiratory syncytial virus infection of epithelial cells. PLoS ONE 15(3):e0230844

Publisher's Note Springer Nature remains neutral with regard to jurisdictional claims in published maps and institutional affiliations. 